\title{
Dynamics of Strongly Coupled Spiking Neurons
}

\author{
Paul C. Bressloff \\ S. Coombes \\ Nonlinear and Complex Systems Group, Department of Mathematical Sciences, \\ Loughborough University, Loughborough, Leicestershire LE11 3TU, U.K.
}

We present a dynamical theory of integrate-and-fire neurons with strong synaptic coupling. We show how phase-locked states that are stable in the weak coupling regime can destabilize as the coupling is increased, leading to states characterized by spatiotemporal variations in the interspike intervals (ISIs). The dynamics is compared with that of a corresponding network of analog neurons in which the outputs of the neurons are taken to be mean firing rates. A fundamental result is that for slow interactions, there is good agreement between the two models (on an appropriately defined timescale). Various examples of desynchronization in the strong coupling regime are presented. First, a globally coupled network of identical neurons with strong inhibitory coupling is shown to exhibit oscillator death in which some of the neurons suppress the activity of others. However, the stability of the synchronous state persists for very large networks and fast synapses. Second, an asymmetric network with a mixture of excitation and inhibition is shown to exhibit periodic bursting patterns. Finally, a one-dimensional network of neurons with long-range interactions is shown to desynchronize to a state with a spatially periodic pattern of mean firing rates across the network. This is modulated by deterministic fluctuations of the instantaneous firing rate whose size is an increasing function of the speed of synaptic response.

\section{Introduction}

There are two basic classes of neurodynamical model that are distinguished by their representation of neuronal output activity (see Abbott, 1994, and references therein). The first considers the output of a neuron to be a mean firing rate that either specifies the number of spikes emitted in some fixed time window (Hopfield, 1984; Amit \& Tsodyks, 1991; Ermentrout, 1994) or corresponds to the probability of firing within some population (Wilson \& Cowan, 1972; Gerstner, 1995). Recently, however, a number of experiments on sensory neurons have shown that the precise timing of spikes may be significant in neuronal information processing, and this has led to renewed interest in the second class of neurodynamical models based on spiking neurons. For example, spike train recordings from $\mathrm{H} 1$, a motion-sensitive 
neuron in the fly visual system, exhibit variability of response to constant stimuli but a high degree of reproducibility for more natural dynamic stimuli (Strong, Koberle, van Steveninck, \& Bialek, 1998). This reproducibility provides an enhanced capacity for carrying information (Rieke, Warland, \& van Steveninck, 1996). Similar findings have been obtained in retinal ganglion cells of the tiger salamander and rabbit (Berry, Warland, \& Meister, 1997). It is also well known that precise spike timing is essential for sound localization in the auditory system of animals such as the barn owl (Carr \& Konishi, 1990). Recent advances in computational neuroscience support the notion that synapses are capable of supporting computations based on highly structured temporal codes (Mainen \& Sejnowski, 1995; Gerstner, Kreiter, Markram, \& Herz, 1997). Moreover, this mode of computation suggests how only one type of neuroarchitecture can support the processing of several very different sensory modalities (Hopfield, 1995). Such codes are undoubtedly important in sensory and cognitive processing. The simplest and most popular example of a spiking neuron is the so-called integrateand-fire (IF) model (Keener, Hoppensteadt, \& Rinzel, 1981; Tuckwell, 1988) and its generalizations (Gerstner, 1995). The state of an IF neuron changes discontinuously (resets) whenever it crosses some threshold and fires, so a complete description in terms of smooth differential equations is no longer possible. This type of model can be derived systematically from more detailed Hodgkin-Huxley equations describing the process of action potential generation (Abbott \& Kepler, 1990; Kistler, Gerstner, \& van Hemmen, 1997).

There are major differences in the analytical treatments of firing-rate and spiking neural network models. A common starting point for the former is to consider conditions under which destabilization of a homogeneous low-activity state occurs, leading to the formation of a state with inhomogeneous and/or time-dependent firing rates (see, for example, Wilson \& Cowan, 1973; Ermentrout \& Cowan, 1979; Atiya \& Baldi, 1989; Li \& Hopfield, 1989; Ermentrout, 1998a). On the other hand, most of the work on IF network dynamics has been concerned with the existence and stability of phase-locked solutions, in which the neurons fire at a fixed common frequency. Analysis of globally coupled IF oscillators in terms of return maps has shown that synchronization almost always occurs in the presence of instantaneous excitatory interactions (Mirollo \& Strogatz, 1990). Subsequently this result has been extended to take into account the effects of inhomogeneities and various synaptic and axonal delays (Treves, 1993; Tsodyks, Mitkov, \& Sompolinsky, 1993; Abbott \& van Vreeswijk, 1993; van Vreeswijk, Abbott, \& Ermentrout, 1994; Ernst, Pawelzik, \& Giesel, 1995; Hansel, Mato, \& Meunier, 1995; Coombes \& Lord, 1997; Bressloff, Coombes, \& De Souza, 1997; Bressloff \& Coombes, 1998, 1999). One finds that for small transmission delays, inhibitory (excitatory) synapses tend to synchronize if the rise time of a synapse is longer (shorter) than the duration of an action potential. Increasing the transmission delay leads to alternating bands of stability and instability of the synchronous state. Analogous results have been ob- 
tained in the spike response model (Gerstner, Ritz, \& van Hemmen, 1993; Gerstner, 1995; Gerstner, van Hemmen, \& Cowan, 1996). Traveling waves of synchronized activity have been investigated in finite chains of IF oscillators modeling locomotion in simple vertebrates (Bressloff \& Coombes, 1998), and in two-dimensional networks where spirals and target patterns are also observed (Chu, Milton, \& Cowan, 1994; Kistler, Seitz, \& van Hemmen, 1998).

In this article we present a theory of spike train dynamics in IF networks that bridges the gap between firing-rate and spiking models. In particular, we show that synaptic interactions that are synchronizing in the weak coupling regime can become desynchronizing for sufficiently strong coupling. The resulting dynamics is compared with the behavior of a corresponding analog model in which the outputs of the neurons are taken to be mean firing rates. A basic result of our work is that for slow interactions, there is good agreement between the two models (on an appropriately defined timescale). On the other hand, discrepancies can arise for fast synapses where IF neurons may remain phase locked.

We take as our starting point the nonlinear mapping of the neuronal firing times and show how a bifurcation analysis of this map can serve as a basis for understanding the extremely rich dynamical structure seen in networks of spiking neurons. Explicit criteria for the stability of phase-locked solutions are derived in both the weak and strong coupling regimes by considering the propagation of perturbations of the firing times throughout a network. In the strong coupling case, the analysis predicts regions in parameter space where instabilities in the firing times may cause transitions to nonphase-locked states. Numerical simulations are used to establish that in these regions, the full nonlinear firing map can support several distinct types of behavior. For small networks, these include mode-locked bursting states, where packets of spikes may be generated, separated by periods of inactivity, and inhomogeneous states in which some of the oscillators become inactive. For large global inhibitory networks with vanishing mean perturbations of the firing times, we show that such bifurcations can be suppressed, in agreement with the mode locking theorem of Gerstner et al. (1996). As a final example of the importance of strong coupling instabilities, we consider a ring of IF neurons with a Mexican hat interaction function. A discrete Turing-Hopf bifurcation of the firing times from a synchronous state to a state with periodic or quasi-periodic variations of the interspike intervals (ISIs) on closed orbits is shown to occur in the strong coupling regime. Further, it is shown how the separation of these orbits in phasespace results in a spatially periodic pattern of mean firing rate across the network.

\section{Integrate-and-Fire Model of a Spiking Neuron}

The standard dynamical system for describing a neuron with spatially constant membrane potential $V$ is based on conservation of electric charge, so 
that

$$
C \frac{\mathrm{d} V}{\mathrm{~d} t}=-F+I_{S}+I
$$

where $C$ is the cell capacitance, $F$ is the membrane current, $I_{S}$ the sum of synaptic currents entering the cell, and $I$ describes any external currents. In the Hodgkin-Huxley model the membrane current arises mainly through the conduction of sodium and potassium ions through voltage-dependent channels in the membrane. The contribution from other ionic currents is assumed to obey Ohm's law. In fact $F$ is considered to be a function of $V$ and of three time-dependent and voltage-dependent conductance variables $m, n$, and $h$,

$$
F(V, m, n, h)=g_{L}\left(V-V_{L}\right)+g_{K} n^{4}\left(V-V_{K}\right)+g_{N a} h m^{3}\left(V-V_{N a}\right),
$$

where $g_{L}, g_{K}$, and $g_{N a}$ are constants and $V_{L}, V_{K}$, and $V_{N a}$ represent the constant membrane reversal potentials associated with the leakage, potassium, and sodium channels, respectively. The conductance variables $m, n$, and $h$ take values between 0 and 1 and approach the asymptotic values $m_{\infty}(V)$, $n_{\infty}(V)$ and $h_{\infty}(V)$ with time constants $\tau_{m}(V), \tau_{n}(V)$ and $\tau_{h}(V)$, respectively.

The conductance-based Hodgkin-Huxley equations depend on four dynamical variables. A reduction of this number is often desirable in order to facilitate any mathematical analysis and to ease the computational burden for simulations of large networks. A systematic approach for doing this involves the use of equivalent potentials (Abbott \& Kepler, 1990; Kepler, Abbott, \& Marder, 1992). Following this approach, one can derive an IF model, which provides a caricature of the capacitative nature of cell membrane at the expense of a detailed model of the refractory process. The IF model satisfies equation 2.1 with $F=F(V)$, together with the condition that whenever the neuron reaches a threshold $h$, it fires, and $V$ is immediately reset to the resting potential $V_{0}$. The dynamics of the membrane conductances $m, n, h$ is eliminated completely. Using a curve-fitting procedure, it is possible to approximate $F(V)$ by a cubic, $F(V)=a\left(V-V_{0}\right)\left(V-V_{1}\right)(V-h)$ where the constants $a, V_{0,1}$, and $h$ can be determined explicitly from the reduction of the underlying Hodgkin-Huxley equations.

At a synapse, presynaptic firing results in the release of neurotransmitters that causes a change in the membrane conductance of the postsynaptic neuron. This postsynaptic current may be written

$$
I_{s}=g_{s} s\left(V_{s}-V\right)
$$

where $V$ is the voltage of the postsynaptic neuron, $V_{s}$ is the membrane reversal potential, and $g_{s}$ is a constant. The variable $s$ corresponds to the probability that a synaptic receptor channel is in an open conducting state. 
This probability depends on the presence and concentration of neurotransmitter released by the presynaptic neuron. Under certain assumptions, it may be shown that a second-order Markov scheme for the synaptic channel kinetics describes the so-called alpha function response commonly used in synaptic modeling (Destexhe, Mainen, \& Sejnowski, 1994): the synaptic response to an incoming spike at time $t_{0}$ is

$$
s(t)=s_{0}\left(t-t_{0}\right) \mathrm{e}^{-\alpha\left(t-t_{0}\right)}, \quad t>t_{0}
$$

for constants $s_{0}, \alpha$. Here $\alpha$ determines the inverse rise time for synaptic response. If we now combine equations 2.1 and 2.3 under the approximation $F=F(V)$, we obtain an equation of the form (after setting $C=1$ )

$$
\frac{\mathrm{d} V}{\mathrm{~d} t}=-F(V)+I+g_{s} \sum_{m} s\left(t-t_{m}\right)\left[V_{s}-V\right] .
$$

We are assuming that the neuron receives a sequence of action potential spikes at times $\left\{t_{m}\right\}$ and each spike generates a synaptic response according to equation 2.4. The neuron itself fires a spike whenever $V(t)$ reaches the threshold $h$, and $V$ is immediately reset to the resting potential, $V_{0}$. Denoting the firing times of the neuron by $\left\{T_{m}\right\}$, we can view the neuron as a device that maps $\left\{t_{m}\right\} \rightarrow\left\{T_{m}\right\}$. We introduce two additional simplifications. First, we neglect shunting effects by setting $V_{s}-V \approx V_{s}$; the possible nonlinear effects of shunting are discussed by Abbott (1991). Second, we reduce $F(V)$ further by considering the linear approximation $F(V)=b\left(V-V_{0}\right)$ (Abbott \& Kepler, 1990). This linear IF model of a spiking neuron will be used in our subsequent analysis of network dynamics (see sections 3 and 4). However, before proceeding further, we briefly mention an alternative formulation of spiking neurons based on the so-called spike response model.

The IF model assumes that it is the capacitative nature of the cell that in conjunction with a simple thresholding process dominates the production of spikes. The spike response (SR) model (Gerstner \& van Hemmen, 1994; Gerstner, 1995; Gerstner et al., 1996) is a more general framework that can accommodate the apparent reduced excitability (or increased threshold) of a neuron after the emission of a spike. Spike reception and spike generation are combined with the use of two separate response functions. The first, $h_{s}(t)$, describes the postsynaptic response to an incoming spike in a similar fashion to the IF model, whereas the second, $h_{r}(t)$, mimics the effect of refractoriness. The refractory function $h_{r}(t)$ can in principle be related to the detailed dynamics underlying the description of ionic channels. In practice, an idealized functional form is often used, although numerical fits to the Hodgkin-Huxley equations during the spiking process are also possible (Kistler et al., 1997). In more detail, a sequence of incoming spikes $\left\{t_{m}\right\}$ evokes a postsynaptic potential in the neuron via $V^{s}(t)=\sum_{m} h_{s}\left(t-t_{m}\right)$, where $h_{s}(t)$ incorporates details of axonal, synaptic, and dendritic processing. The total 
membrane potential of the neuron is taken to be $V(t)=V^{r}(t)+V^{s}(t)$, where $V^{r}(t)=\sum_{m} h_{r}\left(t-T_{m}\right)$ and $\left\{T_{m}\right\}$ is the sequence of output firing times. Whenever $V(t)$ reaches some threshold, the neuron fires a spike, and at the same time a negative contribution $h_{r}$ is added to $V$ so as to approximate the reduced excitability seen after firing. Since the reset condition of the IF model is equivalent to a sequence of current pulses, $-h \sum_{m} \delta\left(T_{m}\right)$, the linear IF model is a special case of the SR model. That is, if $I=0, F(V)=-V$ and we neglect shunting, then we can integrate equation 2.5 to obtain the equivalent formulation

$$
V(t)=\sum_{m} h_{r}\left(t-T_{m}\right)+\sum_{m} h_{s}\left(t-t_{m}\right)
$$

where

$$
h_{r}(t)=-h \mathrm{e}^{-t}, \quad h_{s}(t)=\int_{0}^{t} \mathrm{e}^{-\left(t-t^{\prime}\right)} s\left(t^{\prime}\right) \mathrm{d} t^{\prime}, \quad t>0
$$

and there is no reset.

Most work to date on the analysis of the SR model has been based on the study of large networks using dynamical mean field theory (Gerstner \& van Hemmen, 1994; Gerstner, 1995), which can be viewed as a generalization of the population averaging approach of Wilson and Cowan (1972). This is different from the approach we take here, which is principally concerned with the dynamics of finite networks of spiking neurons. However, we will link the two in section 4.3, where we discuss large, globally coupled networks and the mode-locking theorem of Gerstner et al. (1996).

\section{Averaging Methods for Analyzing IF Network Dynamics}

We now consider a network of IF neurons that interact via synapses by transmitting spike trains to one another. Let $V_{i}(t)$ denote the state of the $i$ th neuron at time $t, i=1, \ldots, N$, where $N$ is the total number of neurons in the network. Suppose that the variables $V_{i}(t)$ evolve according to the equations (cf. equation 2.5)

$$
\frac{\mathrm{d} V_{i}(t)}{\mathrm{d} t}=-\frac{V_{i}(t)}{\tau_{d}}+I_{i}+X_{i}(t)
$$

where $I_{i}$ is a constant external bias, $\tau_{d}$ is the membrane time constant, and $X_{i}(t)$ is the total synaptic current into the cell. We shall fix the units of time by setting $\tau_{d}=1$; typical values for $\tau_{d}$ are in the range 5-20 msec. Equation 3.1 is supplemented by the reset condition that whenever $V_{i}=h$, neuron $i$ fires and is reset to $V_{i}=0$. We shall set the threshold $h=1$. The synaptic current 
$X_{i}(t)$ is generated by the arrival of spikes from neuron $j$ and takes the explicit form

$$
X_{i}(t)=\epsilon \sum_{j=1}^{N} W_{i j} \sum_{m=-\infty}^{\infty} J\left(t-T_{j}^{m}\right),
$$

where $\epsilon W_{i j}$ is the effective synaptic weight of the connection from the $j$ th to the $i$ th neuron, $J(t)$ determines the time course of the postsynaptic response to a single spike with $J(t)=0$ for $t<0$, and $T_{j}^{m}$ denotes the sequence of firing times of the $j$ th neuron with $m$ running through the integers. We have introduced a coupling constant $\epsilon$ characterizing the overall strength of synaptic interactions. From the discussion in section 2, a biologically motivated choice for $J(t)$ is

$$
J(t)=s\left(t-\tau_{a}\right) \Theta\left(t-\tau_{a}\right), \quad s(t)=\alpha^{2} t \mathrm{e}^{-\alpha t},
$$

where $s(t)$ is an alpha function (with unit normalization), $\tau_{a}$ is a discrete axonal transmission delay, and $\Theta$ is the unit step function, $\Theta(t)=1$ if $t>0$ and zero otherwise. The maximum synaptic response then occurs at a nonzero delay $t=\tau_{a}+\alpha^{-1}$. In a companion paper, the effects of dendritic interactions (both passive and active) will be analyzed by taking $J(t)$ to be the Green's function of some cable equation (Bressloff, 1999).

Although equations 3.1 and 3.2 are considerably simpler than those describing a network of Hodgkin-Huxley neurons, the analysis of the resulting dynamics is still a nontrivial problem since one has to handle both the presence of delays and discontinuities arising from reset. Most work to date has therefore been based on some form of approximation scheme. We shall describe two such schemes: phase reduction in the weak coupling regime and time averaging with slow synapses. In section 4 we shall then carry out a direct analysis of the IF network dynamics without recourse to such approximations.

3.1 Weak Coupling: Phase-Oscillator Models. We first show how in the weak coupling limit, equation 3.1 with reset can be reduced to a phaseoscillator equation. For concreteness, assume that $I_{i}=I>1$ for all $i=$ $1, \ldots, N$, so that in the absence of any coupling $(\epsilon=0)$, each neuron acts as a regular oscillator by firing spikes with a constant period $T=\ln [I /(I-1)]$. Following van Vreeswijk et al. (1994), we introduce the phase variable $\psi_{i}(t)$ according to

$$
(\bmod 1) \psi_{i}(t)+\frac{t}{T}=\Psi\left(V_{i}(t)\right) \equiv \frac{1}{T} \int_{0}^{V_{i}(t)} \frac{d x}{F(x)},
$$

where $F(x)=I-x$ for the linear IF model. (One can also apply this transform to the nonlinear IF model discussed in section 2 with $F(x)$ now given by a 
cubic.) Under such a transformation, equation 3.1 becomes

$$
\dot{\psi}_{i}(t)=R_{T}\left(\psi_{i}(t)+t / T\right) X_{i}(t),
$$

with

$$
R_{T}(\theta) \equiv \frac{1}{T} \frac{1}{F\left[\Psi^{-1}(\theta)\right]}=\frac{\left[1-\mathrm{e}^{-T}\right] \mathrm{e}^{T \theta}}{T}
$$

for $0 \leq \theta<1$ and $R_{T}(\theta+k)=R_{T}(\theta)$ for all integers $k$. In the absence of any coupling, the phase variable $\psi_{i}(t)$ is constant in time, and all oscillators fire with their natural periods $T$. For weak coupling, each oscillator still approximately fires at the unperturbed rate, but now the phases slowly drift according to equation 3.5 since $X_{i}(t)=\mathcal{O}(\epsilon)$. To first order in $\epsilon$, we can take the firing times to be $T_{j}^{n}=\left(n-\psi_{j}(t)\right) T$. Under this approximation, equations 3.2 and 3.5 lead to the following equation for the shifted phases $\theta_{i}(t)=\psi_{i}(t)+t / T:$

$$
\frac{\mathrm{d} \theta_{i}}{\mathrm{~d} t}=\frac{1}{T}+\epsilon \sum_{j=1}^{N} W_{i j} R_{T}\left(\theta_{i}\right) P_{T}\left(\theta_{j}\right)+\mathcal{O}\left(\epsilon^{2}\right),
$$

where $P_{T}(\theta+1)=P_{T}(\theta)$ for all $\theta$ and

$$
P_{T}(\theta)=\sum_{m=-\infty}^{\infty} J((\theta+m) T), \quad 0 \leq \theta<1 .
$$

The summation over $m$ in equation 3.8 is easily performed for $J(\tau)$, satisfying equation 3.3, and gives $P_{T}(\theta)=\widehat{J_{T}}\left(\theta-\tau_{a} / T\right)$, where $\widehat{J_{T}}(\theta)$ is a periodic function of $\theta$ with

$$
\widehat{J_{T}}(\theta)=\frac{\alpha^{2} \mathrm{e}^{-\alpha \theta T}}{1-\mathrm{e}^{-\alpha T}}\left[\theta T+\frac{T \mathrm{e}^{-\alpha T}}{1-\mathrm{e}^{-\alpha T}}\right], \quad 0 \leq \theta<1 .
$$

The function $R_{T}$ may be interpreted as the phase-response curve (PRC) of an individual IF oscillator, and $P_{T}$ is the corresponding pulselike function that contains all details concerning the synaptic interactions. Note that phase equations of the form 3.7 can also be derived for a system of weakly coupled limit cycle oscillators based on more detailed biophysical models such as Hodgkin-Huxley neurons (Ermentrout \& Kopell, 1984; Kuramoto, 1984; Hansel et al., 1995). As discussed in some detail by Hansel et al. (1995), linear IF oscillators have a type I PRC, which means that an instantaneous excitatory stimulus always advances its phase $\left(R_{T}(\theta)\right.$ is positive for all $\left.\theta\right)$. On the other hand, Hodgkin-Huxley neurons are of type II since a stimulus can either advance or retard the phase depending on the point on the cycle 
at which the stimulus is applied $\left(R_{T}(\theta)\right.$ takes on both positive and negative values over the domain $\theta \in[0,1])$. Equation 3.7 can be simplified further by averaging over the natural period $T$. This leads to an equation of the form

$$
\frac{\mathrm{d} \theta_{i}}{\mathrm{~d} t}=\omega+\epsilon \sum_{j=1}^{N} W_{i j} H_{T}\left(\theta_{j}-\theta_{i}\right)+\mathcal{O}\left(\epsilon^{2}\right)
$$

with $\omega=1 / T$ and

$$
H_{T}(\phi)=\int_{0}^{1} R_{T}(\theta) P_{T}(\theta+\phi) d \theta .
$$

For positive kernels $J(\tau)$, the interaction function $H_{T}(\phi)$ is positive for all $\phi$ since IF oscillators are of type I. However, it is possible to mimic an effective type II interaction function by introducing a combination of excitatory and inhibitory interactions between the IF neurons in the definition of $J(\tau)$ (Bressloff \& Coombes, 1998a). To illustrate this idea, suppose that we decompose $J(\tau)$ as

$$
\begin{aligned}
& J(\tau)=J_{+}(\tau)-J_{-}(\tau), \quad J_{ \pm}(\tau)=s_{ \pm}\left(\tau-\tau_{ \pm}\right) \Theta\left(\tau-\tau_{ \pm}\right), \\
& s_{ \pm}(\tau)=\alpha_{ \pm}^{2} \tau \mathrm{e}^{-\alpha_{ \pm} \tau},
\end{aligned}
$$

where $J_{ \pm}(\tau)$ represent $\alpha$ functions for excitatory $(+)$ and inhibitory (-) synapses. Assuming that the inhibitory pathways are delayed with respect to the excitatory ones $\left(\tau_{-}>\tau_{+}\right)$then the effective interaction function of an IF oscillator can be approximately sinusoidal. This is illustrated in Figure 1. It is not clear that such a combination of synapses is directly realized in cortical microcircuits. However, it is known that recurrent excitatory connections also stimulate inhibitory interneurons, and this might lead to an effective delay kernel of the form 3.14 at the population level.

We define a phase-locked solution of equation 3.10 to be of the form $\theta_{i}(t)=\phi_{i}+\Omega t$, where $\phi_{i}$ is a constant phase and $\Omega=1 / T+\mathcal{O}(\epsilon)$ is the collective frequency of the coupled oscillators. Substitution of this solution into equation 3.10 and working to $\mathcal{O}(\epsilon)$ leads to the fixed-point equations,

$$
\Omega=\frac{1}{T}+\epsilon \sum_{j=1}^{N} W_{i j} H_{T}\left(\phi_{j}-\phi_{i}\right) .
$$

After choosing some reference oscillator, the $N$ equations (3.13) determine the collective period $\Omega$ and $N-1$ relative phases, with the latter independent of $\epsilon$. In order to analyze the local stability of a phase-locked solution $\Phi_{\sigma_{i}}=$ $\left(\phi_{1}, \ldots, \phi_{N}\right)$, we linearize equation 3.10 by setting $\theta_{i}(t)=\phi_{i}+\Omega t+\widetilde{\theta}_{i}(t)$ and expanding to first order in $\widetilde{\theta}_{i}$ :

$$
\frac{\mathrm{d} \widetilde{\theta}_{i}}{\mathrm{~d} t}=\epsilon \sum_{j=1}^{N} \widehat{\mathcal{H}}_{i j}(\Phi) \widetilde{\theta}_{j},
$$



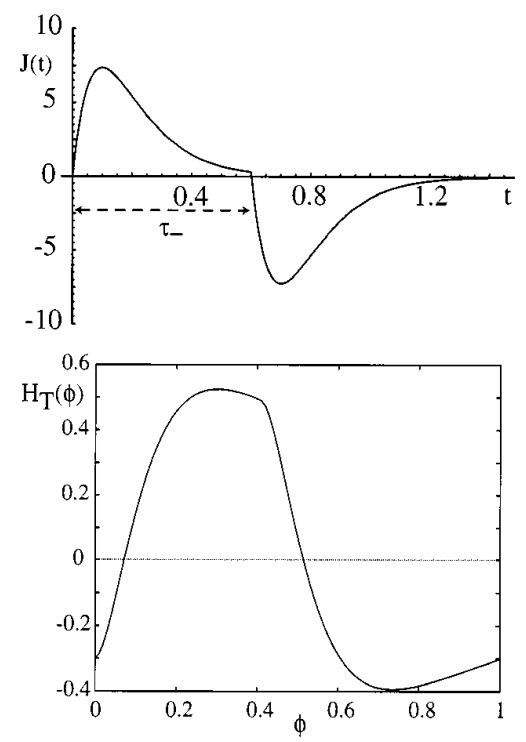

Figure 1: (Top) Delay kernel $J(t)$ and (bottom) associated interaction function $H_{T}(\phi)$ for a combination of excitatory and delayed inhibitory synaptic interactions given by equation 3.12. Here $\alpha_{ \pm}=10, \tau_{+}=0, \tau_{-}=0.6$, and $T=\ln 2$.

where

$$
\widehat{\mathcal{H}}_{i j}(\Phi)=\mathcal{H}_{i j}(\Phi)-\delta_{i, j} \sum_{k=1}^{N} \mathcal{H}_{i k}(\Phi), \quad \mathcal{H}_{i j}(\Phi)=W_{i j} H_{T}^{\prime}\left(\phi_{j}-\phi_{i}\right)
$$

and $H_{T}^{\prime}(\phi)=d H_{T}(\phi) / \mathrm{d} \phi$. One of the eigenvalues of the Jacobian $\widehat{\mathcal{H}}$ is always zero, and the corresponding eigenvector points in the direction of the flow, that is $(1,1, \ldots, 1)$. The phase-locked solution will be stable provided that all other eigenvalues have a negative real part (Ermentrout, 1985).

The existence and stability of phase-locked solutions in the case of a symmetric pair of excitatory or inhibitory IF neurons with synaptic interactions have been studied in some detail by van Vreeswijk et al. (1994). In this case, $N=2$ and $W_{11}=W_{22}=0, W_{12}=W_{21}=1$. Equation 3.13 then shows that the allowed solutions for the relative phase $\phi=\phi_{2}-\phi_{1}$ are given by the zeroes of the odd interaction function $H_{T}^{-}(\phi)=H_{T}(\phi)-H_{T}(-\phi)$. The underlying symmetry of the pair of neurons guarantees the existence of the in-phase or synchronous solution $\phi=0$ and the antiphase or antisynchronous solution $\phi=1 / 2$. Suppose that $\tau_{a}=0$. For small $\alpha$, one finds that the in-phase and antiphase solutions are the only phase-locked solutions. However, as $\alpha$ is increased, a critical value $\alpha_{c}$ is reached, where 

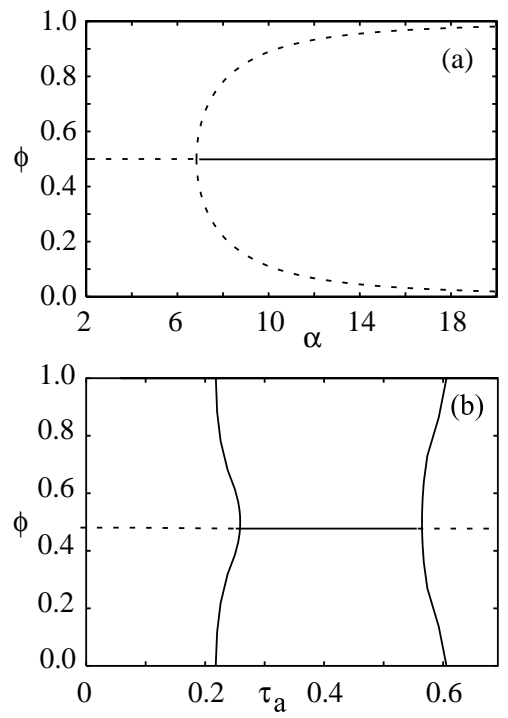

Figure 2: (a) Relative phase $\phi=\phi_{2}-\phi_{1}$ for a pair of IF oscillators with symmetric inhibitory coupling as a function of $\alpha$ with $I=2$. In each case the antiphase state undergoes a bifurcation at a critical value of $\alpha=\alpha_{c}$, where it becomes stable, and two additional unstable solutions $\phi, 1-\phi$ are created. The synchronous state remains stable for all $\alpha$. (b) Relative phase $\phi$ versus discrete delay $\tau_{a}$ for a pair of pulse-coupled IF oscillators with $I=2$ and $\alpha=2$.

there is a bifurcation of the antiphase solution, leading to the creation of two partially synchronized states $\theta$ and $1-\theta$, with $0<\theta<1 / 2$ and $\theta \rightarrow 0$ as $\alpha \rightarrow \infty$ (see Figure 2a and van Vreeswijk et al., 1994). As shown by Coombes and Lord (1997), variation of $\tau_{a}$ for fixed $\alpha$ produces a checkerboard pattern of alternating stable and unstable solution branches (see Figure $2 \mathrm{~b}$ ) that can overlap to produce multistable solutions. Using equation 3.15, one obtains the following necessary and sufficient condition for local asymptotic stability of a phase-locked state in the weak coupling regime:

$$
\epsilon \frac{\mathrm{d} H_{T}^{-}(\phi)}{\mathrm{d} \phi}>0
$$

One finds that for $\tau_{a}=0$ and inhibitory coupling $(\epsilon<0)$, the synchronous state is stable for all $0<\alpha<\infty$. Moreover, the antiphase solution $\phi=1 / 2$ is unstable for $\alpha<\alpha_{c}$, but it gains stability when $\alpha>\alpha_{c}$ with the creation of two unstable partially synchronized states. The stability properties of 


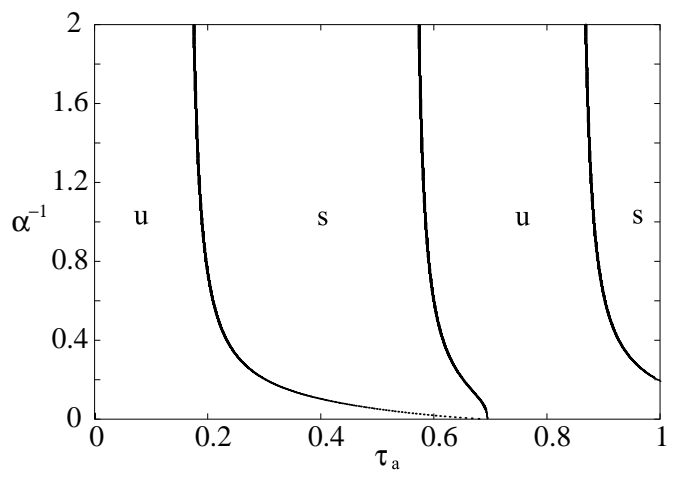

Figure 3: Stability of the synchronous solution $\phi=0$ as a function of $\alpha^{-1}$ and $\tau_{a}$ for weak excitatory coupling with $I=2$. Stable and unstable regions are denoted by $s$ and $u$, respectively. The stability diagrams are periodic in $\tau_{a}$ with period $\ln [I /(I-1)]$. (This periodicity would be distorted in the strong coupling regime since the collective period of oscillation depends on the strength of coupling).

all solutions are reversed in the case of excitatory coupling $(\epsilon>0)$ so that the synchronous state is now unstable for all $\alpha$. If the discrete delay $\tau_{a}$ is increased from zero, then alternating bands of stability and instability are created. An example of such regions is displayed in Figure 3. The corresponding stability diagram for the antisynchronous state may be obtained by shifting the delay according to $\tau_{a} \rightarrow \tau_{a}+T / 2$.

3.2 Slow Synapses: Analog Models. A second approximation scheme for analyzing IF network dynamics is to assume that the synaptic interactions are sufficiently slow so that the output of a neuron can be characterized reasonably well by a mean (time-averaged) firing rate (see, for example, Amit \& Tsodyks, 1991; Ermentrout, 1994). Therefore, let us consider the case in which $J(\tau)$ is given by the alpha function (see equation 3.3) with a synaptic rise time $\alpha^{-1}$ significantly longer than all other timescales in the system. Suppose that the total synaptic current $X_{i}(t)$ to neuron $i$ is described by a slowly varying function of time $t$. If the neuronal dynamics is fast relative to $\alpha^{-1}$, then the actual firing rate $E_{i}(t)$ of a neuron will quickly relax to approximately its steady-state value, that is,

$$
E_{i}(t)=f\left(X_{i}(t)+I_{i}\right),
$$

where, from equation 3.1, the firing-rate function $f$ is of the form

$$
f(X)=\left\{\ln \left[\frac{X}{X-1}\right]\right\}^{-1} \Theta(X-1) .
$$


(Note that we have ignored the effects of absolute refractory period, which is reasonable when the system is operating well below its optimal firing rate). Equation 3.17 relates the dynamics of the firing rate directly to the stimulus dynamics $X_{i}(t)$ through the steady-state response function. In effect, the use of a slowly varying kernel $J(\tau)$ allows a consistent definition of the firing rate so that a dynamical network model can be based on the steady-state properties of an isolated neuron.

Within the above approximation, we can derive a closed set of equations for the synaptic currents $X_{i}(t)$. This is achieved by rewriting equation 3.2 as a pair of differential equations that generate the alpha function $J(t)$ of equation 3.3, and replacing the output spike train of a neuron by the firingrate function (see equation 3.18):

$$
\begin{aligned}
& \alpha^{-1} \frac{\mathrm{d} X_{i}(t)}{\mathrm{d} t}+X_{i}(t)=Y_{i}(t) \\
& \alpha^{-1} \frac{\mathrm{d} Y_{i}(t)}{\mathrm{d} t}+Y_{i}(t)=\epsilon \sum_{j=1}^{N} W_{i j} E_{j}\left(t-\tau_{a}\right) .
\end{aligned}
$$

Here $Y_{i}(t)$ is an auxiliary current.

A common starting point for the analysis of analog models is to consider conditions under which destabilization of a homogeneous low-activity state occurs, leading to the formation of a state with inhomogeneous and/or timedependent firing rates (see Ermentrout, 1998a, for a review). To simplify our analysis, we shall impose the following condition on the external bias $I_{i}$,

$$
I=I_{i}+\epsilon f(I) \sum_{j=1}^{N} W_{i j}
$$

for some fixed $I>1$. Then for sufficiently weak coupling, $|\epsilon| \ll 1$, the ana$\log$ model has a single stable fixed point given by $Y_{i}=X_{i}=\epsilon f(I) \sum_{j} W_{i j}$, such that the firing rates are kept at the value $f(I)$. Suppose that we linearize equations 3.19 and 3.20 about this fixed point and substitute into the linearized equations a solution of the form $\left(X_{k}(t), Y_{k}(t)\right)=\mathrm{e}^{\lambda t}\left(\bar{X}_{k}, \bar{Y}_{k}\right)$. This leads to the eigenvalue equation

$$
\frac{\lambda_{p}^{ \pm}}{\alpha}=-1 \pm \sqrt{\epsilon f^{\prime}(I) v_{p}} \mathrm{e}^{-\lambda_{p}^{ \pm} \tau_{a} / 2}, \quad p=1, \ldots, N,
$$

where $v_{p}, p=1, \ldots, N$, are the eigenvalues of the weight matrix $\mathbf{W}$. The fixed point will be asymptotically stable if and only if $\operatorname{Re} \lambda_{p}^{t}<0$ for all $p$. As $|\epsilon|$ is increased from zero, an instability may occur in at least two distinct ways. If a single real eigenvalue $\lambda$ crosses the origin in the complex $\lambda$-plane, then a static bifurcation can occur, leading to the emergence of additional fixedpoint solutions that correspond to inhomogeneous firing rates. For example, 
if $\epsilon>0$ and $\mathbf{W}$ has real eigenvalues $v_{1}>v_{2}>\cdots>v_{N}$ with $v_{1}>0$, then a static bifurcation will occur at the critical coupling $\epsilon_{c}$ for which $\lambda_{1}^{+}=0$, that is, $1=\sqrt{\epsilon_{c} f^{\prime}(I) v_{1}}$. On the other hand, if a pair of complex conjugate eigenvalues $\lambda=\lambda_{R} \pm i \lambda_{I}$ crosses the imaginary axis $\left(\lambda_{R}=0\right)$ from left to right in the complex plane, then a Hopf bifurcation can occur, leading to the formation of periodic solutions, that is, time-dependent firing rates. For example, suppose that $\tau_{a}=0$ and $\mathbf{W}$ has a pair of complex eigenvalues $\left(\nu, v^{*}\right)$ with $v=r \mathrm{e}^{\mathrm{i} \theta}$ and $0<\theta<\pi$. Denote the corresponding solutions of equation 3.22 by $\left(\lambda_{ \pm}, \lambda_{ \pm}^{*}\right)$. Assuming that all other eigenvalues $\lambda$ have negative real part, a Hopf bifurcation will occur at the critical coupling $\epsilon_{c}$ for which $\operatorname{Re} \lambda_{+}=0$, that is, $1=\sqrt{\epsilon_{c} f^{\prime}(I) r} \cos (\theta / 2)$. An alternative way of generating oscillatory solutions is to have nonzero delays $\tau_{a}$ (Marcus \& Westervelt, 1989). Note that the basic stability results are independent of the inverse rise time $\alpha$.

To illustrate, consider a symmetric pair of analog neurons with inhibitory coupling, $\epsilon<0$, and $W_{11}=W_{22}=0, W_{12}=W_{21}=1$. The weight matrix $\mathbf{W}$ has eigenvalues \pm 1 and eigenmodes $(1, \pm 1)$. Let $x_{i}=X_{i}-\epsilon f(I)$ so that the fixed-point equations become $x_{1}=\epsilon f\left(x_{2}+I\right)-\epsilon f(I)$ and $x_{2}=\epsilon f\left(x_{1}+I\right)-$ $\epsilon f(I)$. One solution is the homogeneous fixed point $x_{i}=0$. A full bifurcation diagram showing the location of the fixed points $x_{1}$ as a function of $|\epsilon|$ is shown in Figure 4 (top). The homogeneous fixed point $x_{i}=0$ is stable for sufficiently small coupling $|\epsilon|$ but destabilizes at the critical point $|\epsilon|=\epsilon_{c}$ with $\epsilon_{c}=1 / f^{\prime}(I)$, where it coalesces with two unstable fixed points. For $|\epsilon|>\epsilon_{c}$, the unstable fixed point at the origin coexists with two stable fixed points (arising from saddle-node bifurcations). Just beyond the bifurcation point, the system jumps from a homogeneous state to a state in which one neuron is active with a constant firing rate $f(I-\epsilon f(I))$ and the other is passive with zero firing rate. Note that a pair of excitatory analog neurons would bifurcate into another homogeneous state. For example, since $I_{i}$ has to decrease to keep the firing rate constant when $\epsilon>0$ (see equation 3.21), for strong enough coupling $I_{i}<1$ so that the quiescent state is also a valid solution.

A well-known result from the analysis of analog neurons is that an excitatory-inhibitory pair can undergo a Hopf bifurcation to an oscillatory solution (Atiya \& Baldi, 1989). As an illustration, consider the case $\epsilon>0$, $\tau_{a}=0$ and $W_{11}=W_{22}=0, W_{12}=-2, W_{21}=1$. Here neuron 2 inhibits neuron 1 , whereas neuron 1 excites neuron 2 . The eigenvalues of the weight matrix are $\pm i$ so that a Hopf bifurcation arises as $\epsilon$ is increased. (See the discussion below equation 3.22.) Let $x_{1}=X_{1}+2 \epsilon f(I)$ and $x_{2}=X_{2}-\epsilon f(I)$ so that there exists a fixed point at $x_{i}=0$. The bifurcation diagram for the amplitude $x_{1}$ as a function of $\epsilon$ is shown in Figure 4 (bottom). It can be seen that the system undergoes a so-called subcritical Hopf bifurcation in which the homogeneous fixed point $x_{i}=0$ becomes unstable and the system jumps to a coexisting stable limit cycle, signaling a solution with periodic firing rates. 

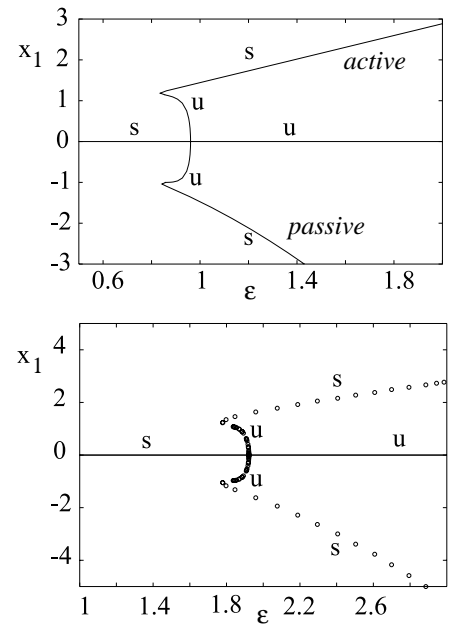

Figure 4: (Top) Bifurcation diagram for a pair of analog neurons with symmetric inhibitory coupling and external input $I=2$. (Bottom) Subcritical Hopf bifurcation for a pair of analog oscillators with self-interactions. $\alpha=0.5, I=2$, $W_{11}=W_{22}=0, W_{21}=1$, and $W_{12}=-2$. Open circles denote the amplitude of the resulting limit cycle from the Hopf bifurcation point $\epsilon \approx 2.0$, and $\mathrm{s}(\mathrm{u})$ stands for stable (unsta ble) dynamics.

This form of jump is often referred to as a hard excitation. The system also exhibits hysteresis. It is interesting to note that if the firing-rate function $f(X)$ of equation 3.18 were taken to be the usual smooth sigmoid function, then, for the given weights, the Hopf bifurcation would be supercritical in the sense that the limit cycle would grow smoothly from the unstable fixed point so that there is no jump phenomenon or hysteresis. This is called a soft excitation. (See Atiya \& Baldi, 1989.) Another important point is that if the analog model were described by a first-order equation rather than a second-order equation (as given by equations 3.19 and 3.20), then it would be necessary to introduce additional self-interactions $\left(W_{12}, W_{21} \neq 0\right)$ in order for a Hopf bifurcation to occur. This is difficult to justify on neurobiological grounds. (A first-order equation would be obtained if the delay kernel were taken to be an exponential function rather than the alpha function 3.3.) We shall return to this issue in section 4.6.

\section{Spike Train Dynamics in the Strong-Coupling Regime}

Recall from section 3 that networks of weakly coupled IF neurons can have stable phase-locked solutions in which all the neurons have the same con- 
stant ISI. On the other hand, a strongly coupled network of analog neurons can bifurcate from a stable homogeneous state with identical timeindependent firing rates to a state with inhomogeneous and/or time-varying firing rates. (Note that a homogeneous state of the analog model does not distinguish between different phase-locked solutions since all phase information is lost during time averaging.) This suggests that there should exist some mechanism for destabilizing phase-locked solutions of the IF model in the strong-coupling regime. Here we identify such a mechanism based on a discrete Hopf bifurcation of the firing times. This induces inhomogeneous and periodic variations in the ISIs, which supports a variety of complex dynamics, including oscillator death (section 4.3), bursting (section 4.4), and pattern formation (section 4.5).

4.1 Phase Locking for Arbitrary Coupling. An important simplifying aspect of the dynamics of pulse-coupled (linear) IF neurons is that it is possible to derive phase-locking equations without the assumption of weak coupling (van Vreeswijk et al., 1994; Bressloff et al., 1997; Bressloff \& Coombes, 1999). This can be achieved by solving equation 3.1 directly under the ansatz that the firing times are of the form $T_{j}^{n}=\left(n-\phi_{j}\right) \bar{T}$ for some self-consistent period $\bar{T}$ and constant phases $\phi_{j}$. Integrating equation 3.1 over the interval $t \in\left(-\bar{T} \phi_{i}, \bar{T}-\bar{T} \phi_{i}\right)$ and incorporating the reset condition by setting $U_{i}\left(-\phi_{i} \bar{T}\right)=0$ and $U_{i}\left(\bar{T}-\phi_{i} \bar{T}\right)=1$ leads to the result

$$
1=\left(1-\mathrm{e}^{-\bar{T}}\right) I_{i}+\epsilon \sum_{j=1}^{N} W_{i j} K_{\bar{T}}\left(\phi_{j}-\phi_{i}\right)
$$

where

$$
K_{T}(\phi)=\mathrm{e}^{-T} \int_{0}^{T} \mathrm{e}^{\tau} \sum_{m=-\infty}^{\infty} J(\tau+(m+\phi) T) d \tau=\frac{T^{2} \mathrm{e}^{-T}}{\left(1-\mathrm{e}^{-T}\right)} H_{T}(\phi) .
$$

Equation 4.1 has an identical structure to that of equation 3.13 and involves the same phase interaction function (up to a multiplicative factor). Indeed, in the weak coupling regime with $I_{i}=I$ for all $i$, equation 4.1 reduces to equation 3.13 with $1 / \bar{T}=\Omega$. This means that techniques previously developed for studying phase locking in weakly coupled oscillator networks can be extended to strongly coupled IF networks. For example, in the case of a ring of identical IF oscillators with symmetric coupling, group-theoretic methods can be used to classify all phase-locked solutions and construct bifurcation diagrams showing how new solution branches emerge via spontaneous symmetry breaking (Bressloff et al., 1997; Bressloff \& Coombes, 1999). Furthermore, in the case of a finite chain of IF oscillators with a gradient of external inputs and sinusoidal-like phase interaction functions of the form shown in Figure 1, one can establish the existence of "traveling 
wave" solutions in which the phase varies monotonically along the chain (except in some narrow boundary layer); such systems can be used to model locomotion in simple vertebrates (Bressloff \& Coombes, 1998a).

There are, however, a number of significant differences between phaselocking equations 3.13 and 4.1. First, equation 4.1 is exact, whereas equation 3.13 is valid only to $\mathcal{O}(\epsilon)$ since it is derived under the assumption of weak coupling. Second, the collective period of oscillations $\bar{T}$ must be determined self-consistently in equation 4.1. Assume for the moment that $\bar{T}$ is given. Suppose that we choose $\theta_{1}$ as a reference oscillator and subtract from equation 4.1 for $i=2, \ldots, N$ the corresponding equation for $i=1$. This leads to $N-1$ fixed-point equations for the $N-1$ relative phases $\hat{\phi}_{j}=\phi_{j}-\phi_{1}$, $j=2, \ldots, N$, which for $I_{i}=I$ take the form

$$
0=\sum_{j=1}^{N} W_{i j} K_{\bar{T}}\left(\hat{\phi}_{j}-\hat{\phi}_{i}\right)-\sum_{j=1}^{N} W_{1 j} K_{\bar{T}}\left(\hat{\phi}_{j}\right),
$$

where $\hat{\phi}_{1} \equiv 0$. The resulting solutions for $\hat{\phi}_{j}, j=2, \ldots, N$, are functions of $\bar{T}$, which can then be substituted back into equation 4.1 for $i=1$ to give an implicit self-consistency condition for $\bar{T}$. The analysis is considerably simpler in the weak-coupling regime since the relative phases are then functions of the natural period $T$. The third difference between weak and strong coupling is that although the equations for phase locking in the two models are formally the same, the underlying dynamical systems are distinct, thus leading to differences in their stability properties. For example, in the special case of a pair of IF neurons, a return map argument can be used to show that equation 3.16 with $T$ replaced by the collective period $\bar{T}$ is a necessary condition for the stability of a phase-locked state for any $\epsilon$ (van Vreeswijk et al., 1994). However, as we shall establish below, it is no longer a sufficient condition for stability in the strong coupling regime. (See also Chow, 1998.)

4.2 Desynchronization in the Strong Coupling Regime. In order to investigate the linear stability of phase-locked solutions of equation 4.1 , we consider perturbations $\delta_{i}^{n}$ of the firing times (van Vreeswijk, 1996; Gerstner et al., 1996; Bressloff \& Coombes, 1999). That is, we set $T_{i}^{n}=\left(n-\phi_{i}\right) \bar{T}+\delta_{i}^{n}$ in equation 3.2 and then integrate equation 3.1 from $T_{i}^{n}$ to $T_{i}^{n+1}$ using the reset condition. This leads to a mapping of the firing times that can be expanded to first order in the perturbations (Bressloff \& Coombes, 1999):

$$
\begin{aligned}
& \left\{I_{i}-1+\epsilon \sum_{j=1}^{N} W_{i j} P_{\bar{T}}\left(\phi_{j}-\phi_{i}\right)\right\}\left[\delta_{i}^{n+1}-\delta_{i}^{n}\right] \\
& =\epsilon \sum_{j=1}^{N} W_{i j} \sum_{m=-\infty}^{\infty} G_{m}\left(\phi_{j}-\phi_{i}, \bar{T}\right)\left[\delta_{j}^{n-m}-\delta_{i}^{n}\right],
\end{aligned}
$$


where $P_{T}$ satisfies equation 3.8 and

$$
G_{m}(\phi, T)=\frac{\mathrm{e}^{-T}}{T} \int_{0}^{T} \mathrm{e}^{t} J^{\prime}(t+(m+\phi) T) d t
$$

The linear delay-difference equation 4.3 has solutions of the form $\delta_{j}^{n}=\mathrm{e}^{n \lambda} \delta_{j}$ with $0 \leq \operatorname{Im}(\lambda)<2 \pi$. The eigenvalues $\lambda$ and eigenvectors $\left(\delta_{1}, \ldots, \delta_{N}\right)$ satisfy the equation

$$
\begin{aligned}
& {\left[I_{i}-1+\epsilon \sum_{j=1}^{N} W_{i j} P_{\bar{T}}\left(\phi_{j}-\phi_{i}\right)\right]\left(\mathrm{e}^{\lambda}-1\right) \delta_{i}} \\
& =\epsilon \sum_{j=1}^{N} W_{i j} G_{\bar{T}}\left(\phi_{j}-\phi_{i}, \lambda\right) \delta_{j}-\epsilon \sum_{j=1}^{N} W_{i j} G_{\bar{T}}\left(\phi_{j}-\phi_{i}, 0\right) \delta_{i},
\end{aligned}
$$

with

$$
G_{T}(\phi, \lambda)=\sum_{m=-\infty}^{\infty} \mathrm{e}^{-m \lambda} G_{m}(\phi, T) .
$$

One solution to equation 4.5 is $\lambda=0$ with $\delta_{i}=\delta$ for all $i=1, \ldots, N$. This reflects the invariance of the dynamics with respect to uniform phase shifts in the firing times, $T_{i}^{n} \rightarrow T_{i}^{n}+\delta$. Thus the condition for linear stability of a phase-locked state is that all remaining solutions $\lambda$ of equation 4.5 have negative real part. This ensures that $\delta_{j}^{n} \rightarrow 0$ as $n \rightarrow \infty$ and, hence, that the phase-locked solution is asymptotically stable. By performing an expansion in powers of the coupling $\epsilon$, it can be established that for sufficiently small coupling, equation 4.5 yields a stability condition that is equivalent to the one based on the Jacobian of the phase-averaged model, equations 3.14 and 3.15. (See Bressloff \& Coombes, 1999.) We wish to determine whether this stability condition breaks down as $|\epsilon|$ is increased (with the sign of $\epsilon$ fixed).

For concreteness, we shall focus on the stability of synchronous solutions. In order to ensure that such solutions exist, we impose the condition

$$
I_{i}=I\left[1-\epsilon K_{T}(0) \sum_{j=1}^{N} W_{i j}\right], i=1, \ldots, N
$$

for some fixed $I>1$ and $T=\ln [I /(I-1)]$. The condition on $I_{i}$ for the IF model plays an analogous role to equation 3.21 for the analog model in section 3.2. The synchronous state $\phi_{i}=\phi$ for all $i$ and arbitrary $\phi$ is then a solution of equation 4.1 with collective period $T$. By fixing $T$ we can make 
a more direct comparison with the analog model whose fixed point at the origin will have a firing rate $f(I)=1 / T$ in equation 3.18. Define the matrix $\widehat{\mathbf{W}}$ according to

$$
\widehat{W}_{i j}=W_{i j}-\delta_{i, j} \sum_{k=1}^{N} W_{i k}
$$

For sufficiently weak coupling, equations 3.14, 3.15, and 4.2 imply that the synchronous state is linearly stable if and only if

$$
\epsilon K_{T}^{\prime}(0) \operatorname{Re}\left[\widehat{v}_{p}\right]<0, p=1, \ldots, N-1,
$$

where $\widehat{v}_{p}, p=1, \ldots, N$ are the eigenvalues of the matrix $\widehat{\mathbf{W}}$ with $\widehat{v}_{N}=0$, and $K_{T}^{\prime}(\theta)=T^{-1} d K_{T}(\theta) / d \theta$. In the particular case of a symmetric pair of neurons, equation 4.9 reduces to the condition $\epsilon K_{T}^{\prime}(0)>0$, which is equivalent to equation 3.16 for $\phi=0$. (The case $\phi \neq 0$ is obtained in exactly the same fashion). It follows that the stability diagram of Figure 3 displays the sign of $K_{T}^{\prime}(0)$ as a function of $\alpha$ and $\tau_{a}$. For example, in the case of zero axonal delays $\left(\tau_{a}=0\right)$, Figure 3 implies that $K_{T}^{\prime}(0)<0$ for all finite $\alpha$ and $T$, and equation 4.9 reduces to the stability condition $\epsilon \operatorname{Re} \widehat{v}_{p}>0$ for all $p=1, \ldots, N-1$.

The stability of the synchronous state for weak coupling implies that the zero eigenvalue is nondegenerate and all other eigenvalues $\lambda$ satisfying equation 4.5 have negative real parts. As the coupling strength $|\epsilon|$ is increased from zero, destabilization of the synchronous state will be signaled by one or more complex conjugate pairs of eigenvalues crossing the imaginary axis in the complex $\lambda$-plane from left to right. (It is simple to establish that the synchronous state cannot destabilize due to a real eigenvalue's crossing the origin by setting $\lambda=0$ in equation 4.5.) We proceed, therefore, by substituting $\phi_{i}=\phi, i=1, \ldots, N$ and imposing the condition 4.7. Equation 4.5 reduces to the form

$$
\left[\left(\mathrm{e}^{\lambda}-1\right)\left(I-1+\epsilon \Gamma_{i} I K_{T}^{\prime}(0)\right)+\epsilon \Gamma_{i} K_{T}^{\prime}(0)\right] \delta_{i}=\epsilon G_{T}(0, \lambda) \sum_{j=1}^{N} W_{i j} \delta_{j}
$$

where $\Gamma_{i}=\sum_{j=1}^{N} W_{i j}$. We have used the identities $P_{T}(0)-I K_{T}(0)=I K_{T}^{\prime}(0)$ and $G_{T}(0,0)=K_{T}^{\prime}(0)$. We then substitute $\lambda=i \beta$ into equation 4.10 and look for the smallest value of the coupling strength, $|\epsilon|=\epsilon_{\mathcal{c}}$, for which a real solution $\beta$ exists. This determines the Hopf bifurcation point at which the synchronous state becomes unstable. (In the special case $\beta=\pi$ this reduces to a period doubling bifurcation.) Note that when $\Gamma_{i}$ is independent of $i$, equation 4.10 can be simplified by choosing $\delta_{i}$ to lie along one of the eigenvectors of the weight matrix $\mathbf{W}$.

We shall explore the nature of the Hopf instability through a number of specific examples. We shall also establish that for slow synapses, the strong 
coupling behavior of the IF model is consistent with that of the mean firingrate model of section 3.2 (on an appropriately defined timescale). In order to compare the two types of model, it will be useful to introduce a few definitions. For a network of $N$ IF neurons labeled $i=1, \ldots, N$, let us define the long-term firing rate of a neuron to be $a_{i}=\bar{\Delta}_{i}^{-1}$, where $\bar{\Delta}_{i}$ is the mean ISI,

$$
\bar{\Delta}_{i}=\lim _{M \rightarrow \infty} \frac{1}{2 M+1} \sum_{m=-M}^{M} \Delta_{i}^{m},
$$

with $\Delta_{i}^{m}=T_{i}^{m+1}-T_{i}^{m}$. A necessary requirement for good agreement between the analog and IF models is that the mean firing rates $a_{i}$ of the IF model match the corresponding firing rates of the analog model. In general, one would also expect to observe fluctuations in the ISIs of the IF network that are not resolved by the analog model. A Hopf bifurcation for maps (also known as a Neimark-Sacker bifurcation) is usually associated with the formation of periodic (or quasiperiodic) orbits, which in the current context corresponds to periodic variations in the ISIs. In cases where the analog model bifurcates to a state with time-independent firing rates, we expect the periodic orbits of the ISIs to be small relative to the firing period, at least for slow synapses; that is, $\left|\Delta_{i}^{m}-\bar{\Delta}_{i}\right| / \bar{\Delta}_{i} \ll 1$ for all $i, m$. (See the example of pattern formation in section 4.5, in particular, Figure 12.) On the other hand, in cases where an analog network destabilizes to a state with time-varying firing rates, we expect the periodic orbits of the ISIs in the IF model to be relatively large. Under such circumstances, we can introduce a sliding window of width $2 P+1$ for the IF model in order to define a short-term average firing rate of the form $a_{i}(m)=\bar{\Delta}_{i}(m)^{-1}$ where

$$
\bar{\Delta}_{i}(m)=\frac{1}{2 P+1} \sum_{p=-P}^{P} \Delta_{i}^{m+p}
$$

One can then see if there is a good match between the time-dependent firing rates of the two models for an appropriately chosen value of $P$. Actually, in practice it is simpler to compare variations in the firing rate of an analog network directly with variations in the ISIs of the corresponding IF network (see Figure 8).

4.3 Oscillator Death in a Globally Coupled Inhibitory Network. As our first example illustrating the Hopf instability identified in section 4.2, we consider a network of $N$ identical IF oscillators with all-to-all inhibitory coupling and no self-interactions. This type of architecture has been used, for example, to model the reticular thalamic nucleus (RTN), which is thought to act as a pacemaker for synchronous spindle oscillations observed during sleep or anesthesia (Wang \& Rinzel, 1992; Golomb \& Rinzel, 1994). In the 
biophysical model of RTN developed by Wang and Rinzel (1992), neural oscillations are sustained by postinhibitory rebound, a phenomenon whereby a neuron can fire after being hyperpolarized over an extended period and then released. This should be contrasted with our simple IF model in which oscillations are sustained by an external bias. We shall show that for slow synapses, desynchronization via a Hopf bifurcation in the firing times occurs, leading to oscillator death in the strong coupling regime, that is, certain cells suppress the activity of others. (See also the recent study of mutually inhibitory Hodgkin-Huxley neurons by White, Chow, Ritt, Soto-Trevino, \& Kopell, 1998.)

The weight matrix $\mathbf{W}$ of a globally coupled inhibitory network with $\epsilon<0$ is given by $W_{i i}=0$ and $W_{i j}=1 /(N-1)$ so that $\Gamma_{i}=1$ for all $i=1, \ldots, N$. It follows that $\mathbf{W}$ has a nondegenerate eigenvalue $v_{+}=1$ with corresponding eigenvector $(1,1, \ldots, 1)$ and an $(N-1)$-fold degenerate eigenvalue $v_{-}=-1 /(N-1)$. The eigenvalues of the matrix $\widehat{\mathbf{W}}$, equation 4.8 , are $\widehat{v}_{+}=0$ and $\widehat{v}_{-}=-N /(N-1)$, so that the synchronous state is stable in the weak coupling regime provided that $\epsilon K_{T}^{\prime}(0)>0$ (see equation 4.9). This is certainly true for zero axonal delays (see Figure 3 ). Note that the underlying permutation symmetry of the system means that there are additional phase-locked states in which the neurons are divided into two or more clusters of synchronized cells (Golomb \& Rinzel, 1994). We shall investigate the stability of the synchronous state as $|\epsilon|$ is increased. Take $\left(\delta_{1}, \ldots, \delta_{N}\right)$ in equation 4.10 to be an eigenvector corresponding to either $v=v_{+}$or $v=v_{-}$ and set $\lambda=i \beta$. Equating real and imaginary parts then leads to the pair of equations,

$$
\begin{aligned}
\epsilon K_{T}^{\prime}(0)+[\cos (\beta)-1]\left[I-1+\epsilon I K_{T}^{\prime}(0)\right] & =\epsilon C(\beta) v \\
\sin (\beta)\left[I-1+\epsilon I K_{T}^{\prime}(0)\right] & =-\epsilon S(\beta) v,
\end{aligned}
$$

where $C(\beta)=\operatorname{Re} G_{T}(0, i \beta),, S(\beta)=-\operatorname{Im}_{T}(0, i \beta)$.

In Figure 5 we plot the solutions $\epsilon$ of equation 4.12 as a function of the inverse rise time $\alpha$ for a pair of inhibitory neurons $(N=2)$ with $T=\ln 2$ and $\tau_{a}=0$. The solid (dashed) solution branch corresponds to the eigenvalue $v=-1(v=1)$. The lower branch determines the critical coupling $|\epsilon|=\epsilon_{c}(\alpha)$ for a Hopf instability. An important result that emerges from this figure is that there exists a critical value $\alpha_{0}$ of the inverse rise time beyond which a Hopf bifurcation cannot occur. That is, if $\alpha>\alpha_{0}$, then the synchronous state remains stable for arbitrarily large inhibitory coupling. On the other hand, for $\alpha<\alpha_{0}$, destabilization of the synchronous state occurs as the coupling strength $\epsilon$ crosses the solid curve of Figure 5 from below. This signals the activation of the eigenmode $\left(\delta_{1}, \delta_{2}\right)=(1,-1)$, which suggests that an inhomogeneous state will be generated. Indeed, direct numerical simulation of the IF model shows that just after destabilization of the synchronous state $\left(|\epsilon|>\epsilon_{c}\right)$, the system jumps to an inhomogeneous state consisting of one active neuron and one passive neuron. We conclude 


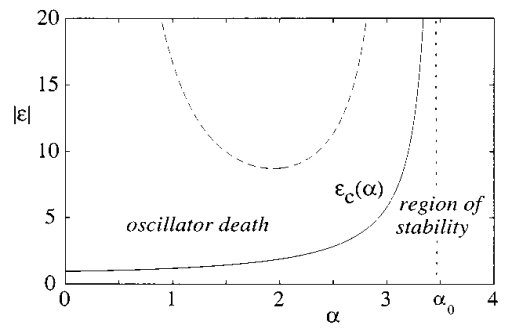

Figure 5: Region of stability for a synchronized pair of identical IF neurons with inhibitory coupling and collective period $T=\ln 2$. The solid curve $|\epsilon|=$ $\epsilon_{c}(\alpha)$ denotes the boundary of the stability region, which is obtained by solving equation 4.12 for $v=-1$ as a function of $\alpha$ with $\tau_{a}=0$. Crossing the boundary from below signals excitation of the linear mode $(1,-1)$, leading to a stable state in which one neuron becomes quiescent (oscillator death). For $\alpha>\alpha_{0}$, the synchronous state is stable for all $\epsilon$. The dashed curve denotes the solution of equation 4.12 for $v=1$.

that for sufficiently slow synapses, a pair of IF neurons displays similar behavior to a corresponding pair of analog neurons in the strong coupling regime (see section 3.2 and Figure 4 (top)). A rough order estimate for the critical inverse rise time $\alpha_{0}$ is $\alpha_{0}^{-1} \approx T$, where $T$ is the collective period of oscillations before destabilization. Such a result is not surprising since one would expect that for a reasonable match between the IF and (timeaveraged) analog models to occur, the IF neurons should sample incoming spike trains over a sliding window that is not too small. The width of such a sliding window is determined by the rise time $\alpha^{-1}$.

The above result appears to be quite general. For example, suppose that we have a nonzero discrete delay $\tau_{a}$ such that the synchronous state is unstable and the antiphase state is stable for a given $\alpha$. The latter state also destabilizes via a Hopf bifurcation in the firing times for small $\alpha$, leading to oscillator death. The result extends to larger values of $N$, for which $v_{-}=-1 /(N-1)$ in equation 4.12. Here desynchronization of a phase-locked state leads to clusters of active and passive neurons. Interestingly, the critical value $\alpha_{0}$ decreases with $N$, indicating the greater tendency for phase locking to occur in large, globally coupled networks. We plot $\epsilon_{c}$ as a function of $\alpha$ for a range of values of network size $N$ in Figure 6, where it can be seen that $\alpha_{0}(N) \rightarrow 0$ as $N \rightarrow \infty$. This implies that for large networks, the neurons remain synchronized for arbitrarily large coupling, even for slow synapses. Indeed, since real neurons typically have an inverse rise time $\alpha$ of the order 2 or larger, it follows that for realistic synaptic time constants, the network can never experience oscillator death for $N$ larger than 5 or so. (There is one subtlety to be noted here. The dashed solution curve shown 


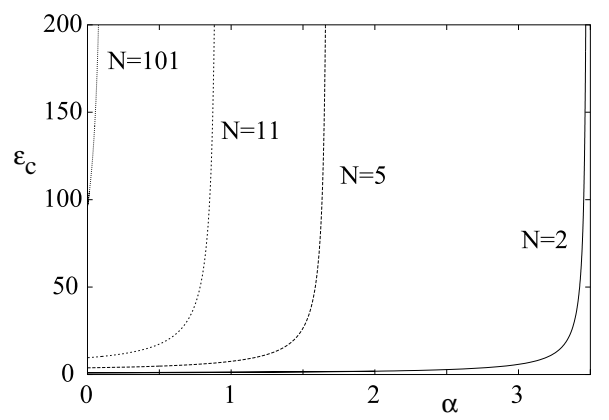

Figure 6: Plot of critical coupling $\epsilon_{c}$ as a function of $\alpha$ for various network sizes $N$. The critical inverse rise time $\alpha_{0}(N)$ is seen to be a decreasing function of $N$, with $\alpha_{0}(N) \rightarrow 0$ as $N \rightarrow \infty$.

in Figure 5 corresponds to excitation of the uniform mode $(1,1, \ldots, 1)$ and is independent of $N$. As $N$ increases, it is crossed by the curve $\epsilon_{c}(\alpha)$ so that for a certain range of values of $\alpha$, it is possible for the synchronous state to destabilize due to excitation of the uniform mode. The neurons in the new state will still be synchronized, but the spike trains will no longer have a simple periodic structure.)

The persistence of synchrony in large networks is consistent with the mode-locking theorem obtained by Gerstner et al. (1996) in their analysis of the spike response model. We shall briefly discuss their result within the context of the IF model. For the given globally coupled network, the linearized map of the firing times, equation 4.3, becomes

$$
\begin{aligned}
& \left\{I-1+\epsilon I K_{T}^{\prime}(0)\right\}\left[\delta_{i}^{n+1}-\delta_{i}^{n}\right] \\
& =\epsilon \sum_{m=-\infty}^{\infty} G_{m}(0, T)\left[\frac{1}{N-1} \sum_{j \neq i} \delta_{j}^{n-m}-\delta_{i}^{n}\right] .
\end{aligned}
$$

The major assumption underlying the analysis of Gerstner et al. (1996) is that for large $N$, the mean perturbation $\left\langle\delta^{m}\right\rangle=\sum_{j \neq i} \delta_{j}^{m} /(N-1) \approx 0$ for all $m \geq 0$ say. Equation 4.13 then simplifies to the one-dimensional, first-order mapping,

$$
\delta_{i}^{n+1}=\frac{I-1+\epsilon(I-1) K_{T}^{\prime}(0)}{I-1+\epsilon I K_{T}^{\prime}(0)} \delta_{i}^{n} \equiv k_{T} \delta_{i}^{n} .
$$

The synchronous (coherent) state will be stable if and only if $\left|k_{T}\right|<1$. It is instructive to establish explicitly that equation 4.14 is equivalent to the 
corresponding result derived for the spike-response model (see equation 4.8 of Gerstner et al., 1996), which in our notation can be written as

$$
\delta_{i}^{n+1}=\frac{\sum_{l \geq 1} h_{r}^{\prime}(l T) \delta_{i}^{n+1-l}}{\sum_{l \geq 1} h_{r}^{\prime}(l T)+\epsilon \sum_{l \geq 1} h_{s}^{\prime}(l T)} .
$$

Here $h_{r}^{\prime}(t)=d h_{r}(t) / d t$. First, using equations 2.7,3.3, and 4.2, it can be shown that $\sum_{l \geq 1} h_{r}^{\prime}(l T)=\mathrm{e}^{-T} /\left(1-\mathrm{e}^{-T}\right)=I-1$ and $\sum_{l \geq 1} h_{s}^{\prime}(l T)=I K_{T}^{\prime}(0)$. Setting $A(T)=I-1+\epsilon I K_{T}(0)$, we can then rewrite equation 4.15 as $A(T) \delta_{i}^{n+1}=$ $\sum_{l>1} \mathrm{e}^{-l T} \delta_{i}^{n+1-l}$. It follows that $A(T) \delta_{i}^{n+1}-\mathrm{e}^{-T} A(T) \delta_{i}^{n}=\mathrm{e}^{-T} \delta_{i}^{n}$, which is identical to equation 4.14 since $\mathrm{e}^{-T}=(I-1) / I$. Equation 4.15 or equation 4.14 implies that the synchronous state is stable in the large $N$ limit provided that $\epsilon \sum_{l>1} h_{s}^{\prime}(l T)>0$, that is, $\epsilon K_{T}^{\prime}(0)>0$ (cf. equation 3.16). This is the essence of the mode-locking theorem of Gerstner et al. (1996). Our analysis also highlights one of the simplifying features of the IF model with alpha function response kernels: that the summations over $l$ in equation 4.15 can be performed explicitly. It would be of interest, however, to investigate to what extent the results of this article carry over to more general choices of $h_{r}$ and $h_{s}$ in the construction of the spike-response model. We expect that the inclusion of details concerning refractoriness will not alter the basic picture, for oscillator death is also found in a pair of mutually inhibitory HodgkinHuxley neurons, as shown by White et al. (1998) in the case of synapses with first-order channel kinetics, and also confirmed numerically by ourselves in the case of second-order kinetics.

Finally, in other related work, van Vreeswijk (1996) has shown how a network of IF neurons with global excitatory coupling can destabilize from an asynchronous state via a Hopf bifurcation in the firing times. However, this leads to small-amplitude quasiperiodic variations in the ISIs of the oscillators (the ISIs lie on relatively small, closed orbits). This can be understood by looking at a corresponding network of excitatory analog neurons, which can bifurcate only to another homogeneous time-independent state.

4.4 Bursting in an Excitatory-Inhibitory Pair of IF Neurons. Now let us consider an excitatory-inhibitory pair of IF neurons with $\epsilon>0, W_{11}=$ $W_{22}=0$ and $W_{12}=-2, W_{21}=1$ and zero axonal delays $\tau_{a}=0$. The analog version of this network, studied in section 3.2, was shown to exhibit periodic variations in the mean firing rate in the strong coupling regime. It can be established from equation 4.9 that the synchronous state is stable for sufficiently weak coupling. In order to investigate Hopf instabilities in the strong coupling regime, we set $\lambda=i \beta$ in equation 4.10 and solve for $(\epsilon, \beta)$ as a function of $\alpha$. For each $\alpha$, the smallest solution $\epsilon=\epsilon_{\mathcal{C}}$ determines the Hopf bifurcation point, which leads to a stability diagram of the form shown in Figure 7. In contrast to the previous example, a critical coupling for a Hopf bifurcation in the firing times exists for all $\alpha$. Direct numerical solution of the system shows that beyond the Hopf bifurcation point, the 


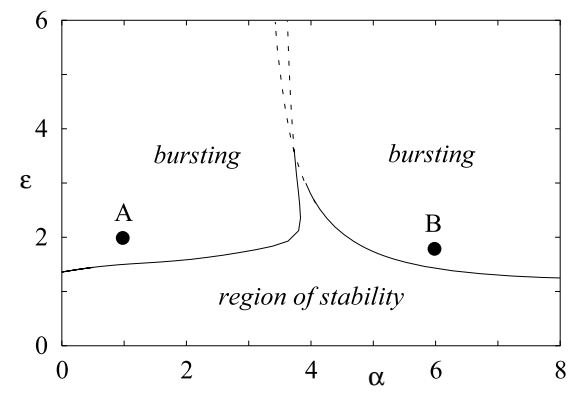

Figure 7: Region of stability for an excitatory-inhibitory pair of IF neurons with inhibitory self-interactions, $\epsilon>0$ and $W_{11}=W_{22}=0, W_{21}=1, W_{12}=-2$. The collective period of synchronized oscillations is taken to be $T=\ln 2$. Solid and dashed curves denote solutions of equation 4.10 for $\epsilon$ with $\lambda=i \beta$, real $\beta$. Crossing the solid boundary of the stability region from below signals destabilization of the synchronous state, leading to the formation of a periodic bursting state.

system jumps to a state in which the two neurons exhibit periodic bursting patterns (see Figure 8). This can be understood in terms of mode-locking associated with periodic variations of the ISIs on closed attracting orbits (see Figure 9). Suppose that the $k$ th oscillator has a periodic solution of length $M_{k}$ so that $\Delta_{k}^{n+p M_{k}}=\Delta_{k}^{n}$ for all integers $p$. If $\Delta_{k}^{1} \gg \Delta_{k}^{n}$ for all $n=2, \ldots, M_{k}$, say, then the resulting spike train exhibits bursting with the interburst interval equal to $\Delta_{k}^{1}$ and the number of spikes per burst equal to $M_{k}$. Although both oscillators have different interburst intervals $\left(\Delta_{1}^{1} \neq \Delta_{2}^{1}\right)$ and numbers of spikes per burst $\left(M_{1} \neq M_{2}\right)$, their spike trains have the same total period, that is, $\sum_{n=1}^{M_{1}} \Delta_{1}^{n}=\sum_{n=1}^{M_{2}} \Delta_{2}^{n}$. Due to the periodicity of the activity, the ISIs fall on only a number of discrete points on the orbit, which is radically different from the case found in van Vreeswijk (1996), where the whole curve is visited due to the quasiperiodical nature of the firing. (Quasiperiodicity is also observed in the pattern formation example presented in section 4.5.) The variation of the ISIs $\Delta_{i}^{n}$ with $n$ is compared directly with the corresponding variation of the firing rates of the analog model in Figure 8. Good agreement can be seen between the two models in the case of small $\alpha$ (see Figure $8 \mathrm{a}$ ), but discrepancies between the two arise as $\alpha$ increases (see Figure 8b). As in the case of oscillator death, the occurrence of bursting through a Hopf bifurcation in the firing times appears to be quite general. For example, suppose that we have a nonzero discrete delay $\tau_{a}$ such that the synchronous state is unstable and the antiphase state is stable for a given $\alpha$. We find that the latter state also destabilizes via a Hopf bifurcation in the firing times for small $\alpha$, leading to a periodic bursting state. 
(a)

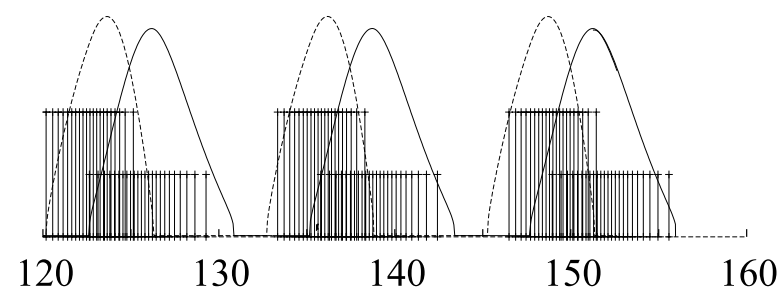

(b)

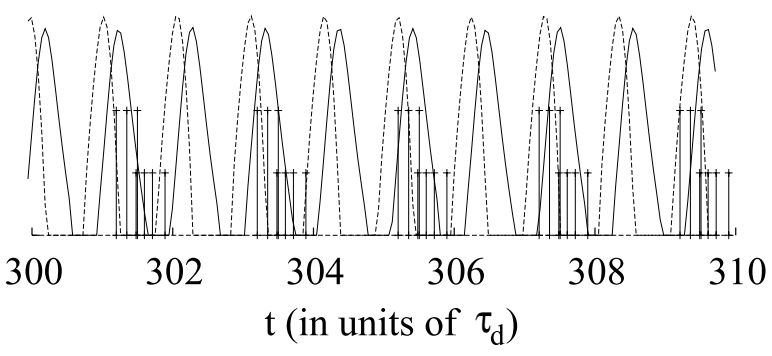

Figure 8: Spike train dynamics for a pair of IF neurons with both excitatory and inhibitory coupling corresponding to points $A$ and $B$ in Figure 7, respectively. The firing times of the two oscillators are represented with lines of different heights (marked with a +). Smooth curves represent variation of firing rate in the analog version of the model.

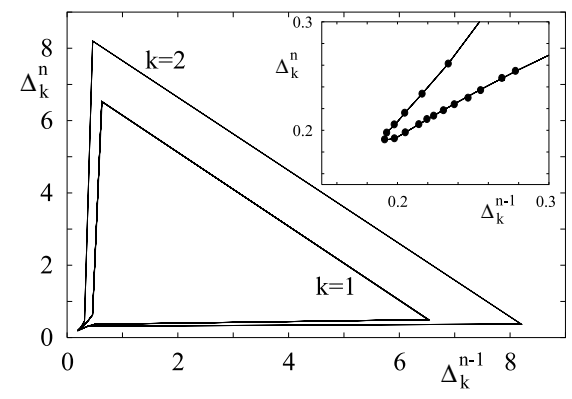

Figure 9: A plot of the ISIs $\left(\Delta_{k}^{n-1}, \Delta_{k}^{n}\right)$ of the spike trains shown in Figure 8a, illustrating how they lie on closed periodic orbits. Points on an orbit are connected by lines. Each triangular region is associated with only one of the neurons, highlighting the difference in interburst intervals (see also Figure 8a). The inset is a blowup of orbit points for one of the neurons within a burst. 


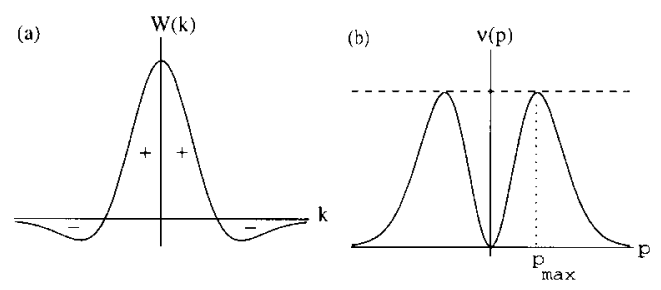

Figure 10: (a) Example of a Mexican hat function weight distribution $W(k)$ and (b) its eigenvalue distribution $v(p)$.

Our analysis of a pair of excitatory-inhibitory IF neurons suggests that bursting can arise at a network level due to strong synaptic coupling without the need for additional slow ionic currents (see Wang \& Rinzel, 1995). An alternative mechanism for generating bursts in networks of interacting neurons is based on electrical (diffusive) coupling between cells. This has been studied in small networks of leaky-integrator neurons with postinhibitory rebound (Mulloney, Perkel, \& Budelli, 1981) and in large networks of Hodgkin-Huxley neurons (Han, Kurrer, \& Kuramoto, 1995). Our analysis also shows that bursting can occur in the absence of the self-interaction terms considered in our previous work (Bressloff \& Coombes, 1998b); it is hard to justify the presence of such terms on neurophysiological grounds. (See section 4.6.)

4.5 Pattern Formation in a One-Dimensional Network. As our final example of strong-coupling Hopf instabilities in IF networks, we consider a ring of $N=2 M+1$ neurons evolving according to equations 3.1 and 3.2 with $\epsilon>0, \tau_{a}=0$ and a weight matrix $W_{i j}=W(i-j)$ where

$$
W(k)=A_{1} \exp \left(-k^{2} /\left(2 \sigma_{1}^{2}\right)\right)-A_{2} \exp \left(-k^{2} /\left(2 \sigma_{2}^{2}\right)\right), \quad 0<k \leq M .
$$

$W(k)=0$ for $|k|>M$ and $W(0)=0$ (no self-interactions). We choose $A_{1}>A_{2}$ and $\sigma_{1}<\sigma_{2}$. W $(k)$ then represents a lattice version of a Mexican hat interaction function in which there is competition between short-range excitation and long-range inhibition. A continuum version of $W(k)$ is plotted in Figure 10a for illustration. This type of architecture is well known to support spatial pattern formation in analog neural systems (Ermentrout \& Cowan, 1979; Ermentrout, 1998a). We shall show that similar behavior occurs in the IF model.

For the given weight matrix $\mathbf{W}$ and homogeneous external inputs $I_{i}=I$, $i=1, \ldots, N$, the network is translationally invariant. This means that one class of solution to the phase-locking equations 4.1 consists of "traveling waves" of the form $\phi_{k}=q k / N$ for integers $q=0, \ldots, N-1$. This type of phase-locked solution also arises in studies of the spike-response model 
(Kistler et al., 1998) and in studies of weakly coupled phase oscillators (Crook, Ermentrout, Vanier, \& Bower, 1997; Bressloff \& Coombes, 1997). We shall concentrate on the synchronous solution $q=0$. For convenience, we shall assume that there is an equal balance between excitation and inhibition by fixing $A_{1}, A_{2}$ in equation 4.16 so that $\Gamma_{i} \equiv \sum_{j=1}^{N} W_{i j}=\sum_{k=-M}^{M} W(k)=0$ for all $i$. (This condition is not necessary for pattern formation to occur.) The collective period of synchronous oscillations is then $T=\ln [I /(I-1)]$ (see equation 4.7.) In order to investigate the linear stability of the synchronous state, we need to determine the eigenvalues of the weight matrix $\mathbf{W}$. These are given by

$$
v(p)=2 \sum_{k=1}^{M} W(k) \cos (p k), \quad p=0, \frac{2 \pi}{N}, \ldots, \frac{2 \pi(N-1)}{N},
$$

with $v(p)>v(0)=0$ for all $p \neq 0$. The corresponding eigenvectors are $\delta_{k}=\mathrm{e}^{i k p}$. Equation 4.9 then shows that the synchronous state is stable in the weak coupling regime, since $K_{T}^{\prime}(0)<0$ for all $\alpha$ (when $\tau_{a}=0$ ) and $\widehat{v}(p)=$ $v(p)$. Let $\pm p_{\max }$ be the wave numbers at which $v(p)$ attains its maxima. (This is illustrated for the continuum Mexican hat function in Figure 10b.) Take $\delta_{k}=\mathrm{e}^{i k p}$ and $\Gamma_{i}=0$, and set $\lambda=i \beta$ in equation 4.10. Equating real and imaginary parts leads to the pair of equations

$$
\begin{aligned}
{[\cos (\beta)-1][I-1] } & =\widehat{\epsilon} C(\beta) \\
\sin (\beta)[I-1] & =-\widehat{\epsilon} S(\beta),
\end{aligned}
$$

where $\widehat{\epsilon}=\epsilon v_{\max }$ and $v_{\max }=v\left(p_{\max }\right)$. We find that there exist non-trivial solutions of equation 4.18 for all values of $\alpha$ (see Figure 11). It follows that a Hopf bifurcation occurs due to activation of the eigenmodes $\delta_{k}=\mathrm{e}^{ \pm i p_{\max } k}$. Since $p_{\max } \neq 0$, the instability will involve spatially periodic perturbations of the firing times, and this leads to the formation of regular activity patterns across the network, as illustrated in Figure 12.

When the network undergoes a Hopf bifurcation, it leads to the creation of periodic orbits for the ISIs. Define the long-term average firing rate according to $a_{k}=\bar{\Delta}_{k}^{-1}$, where $\bar{\Delta}_{k}$ is defined in equation 4.11 . It follows that spatial ( $k$-dependent) variations in the firing rates $a_{k}$ can occur if there is a corresponding spatial distribution of the orbits in phase-space. Figure 12 shows that the orbits are indeed separated from each other in phase-space (although they remain small relative to the natural timescales of the system). The resulting long-term average behavior of the system is characterized by spatially regular patterns of output activity as shown in the inset of Figure 12. These patterns are found to be consistent with those observed in the corresponding analog version of the model presented in section 3.2, and such agreement holds over a wide range of values of $\alpha$ that includes both fast and slow synapses. However, the IF model has additional fine structure 


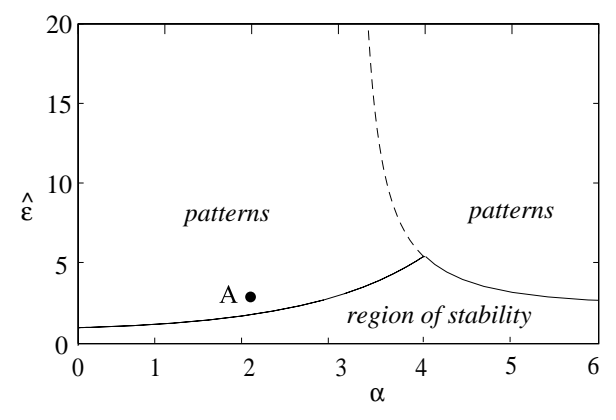

Figure 11: Region of stability for a ring of $N=51 \mathrm{IF}$ neurons having the weight distribution $W(k)$ of equation 4.16 with $\sigma_{1}=2.1, \sigma_{2}=3.5, A_{1}=1.77$, and $\sum_{k} W(k)=0$. The collective period of synchronized oscillations is taken to be $T=\ln 1.5$. Solid and dashed curves denote solutions of equation 4.18 for $\widehat{\epsilon}$. Crossing the solid boundary of the stability region from below signals destabilization of the synchronous state, leading to the formation of spatially periodic patterns of activity as shown in Figure 12.

associated with the dynamics on the periodic orbits of Figure 12, which is not resolved by the analog model. This is illustrated in the insets of Figure 13, where we plot temporal variations in the inverse ISI (or instantaneous firing rate) of a single oscillator for two different values of $\alpha$. It can be seen that there are deterministic fluctuations in the mean firing rate. In order to characterize the size of these fluctuations, we define a deterministic coefficient of variation $C_{V}(k)$ for a neuron $k$ according to

$$
C_{V}(k)=\frac{\sqrt{\overline{\left(\Delta_{k}-\bar{\Delta}_{k}\right)^{2}}}}{\bar{\Delta}_{k}},
$$

with averages defined by equation 4.12 for some sliding window of width $P$. The $C_{V}$ for a single neuron is plotted as a function of $\alpha$ in Figure 13. This shows that the relative size of the deterministic fluctuations in the mean firing rate is an increasing function of $\alpha$. For slow synapses $(\alpha \rightarrow 0)$, the $C_{V}$ is very small, indicating an excellent match between the IF and analog models. However, the fluctuations become more significant when the synapses are fast. For IF networks with a type of disordered Mexican hat interaction, instantaneous synapses, and stochastic external input, it is also known that a further component to the $C_{V}$ arises from the amplification of correlated fluctuations (Usher, Stemmler, Koch, \& Olami, 1994). Interestingly Softy and Koch (1992) have shown that stochastic input alone cannot account for the high ISI variability exhibited by cortical neurons in awake monkeys. 


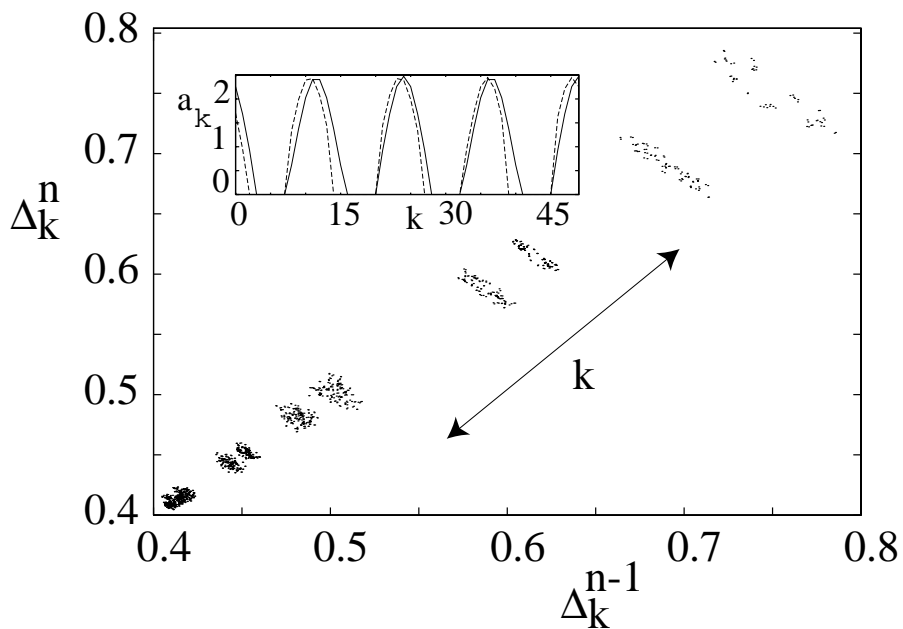

Figure 12: Separation of the ISI orbits in phase-space for a ring of $N=51$ IF corresponding to point $A$ in Figure $11(\alpha=2, \epsilon=0.4)$. The attractor of the embedded ISI with coordinates, $\left(\Delta_{k}^{n-1}, \Delta_{k}^{n}\right)$, is shown for all $N$ neurons. (Inset) Regular spatial variations in the long-term average firing rate $a_{k}$ (dashed curve) are in good agreement with the corresponding activity pattern (solid curve) found in the analog version of the network constructed in section 3.2, with $a_{k}$ now determined by the mean firing-rate function (see equation 3.18).

One potential application of the above analysis is to the study of an IF model of orientation selectivity in simple cells of cat visual cortex. Such a model has been investigated numerically by Somers, Nelson, and Sur (1996), who consider a ring of IF neurons with $k$ labeling the orientation preference $\theta=\pi k / N$ of a given neuron. The neurons interacted via synapses with spike-evoked conductance changes described by alpha functions and with a pattern of connectivity consisting of short-range excitation and long-range inhibition analogous to the Mexican hat function of Figure 10. Numerical investigation showed how the recurrent interactions led to sharpening of orientation tuning curves. Note that the model of Somers et al. (1996) also included shunting terms and time-dependent thresholds; it should be possible to extend the analysis of our article to account for such features.

4.6 Bursting and Oscillator Death Revisited. In section 3.2 we pointed out that in the case of a first-order analog model, an excitatory-inhibitory pair of neurons without self-interactions cannot undergo a Hopf bifurcation. This suggests that the bursting behavior studied in section 4.4 might 


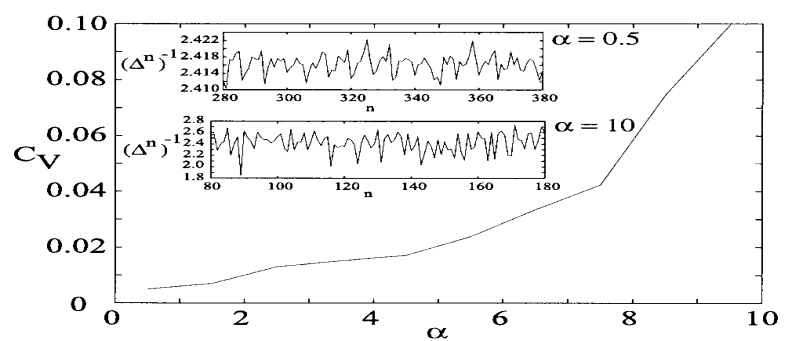

Figure 13: Plot of the coefficient of variation $C_{V}$ for a single IF oscillator as a function of the inverse rise time $\alpha$. All other parameter values are as in Figure 12. For each $\alpha$, the oscillator is chosen to be one with the maximum mean firing rate. (Insets) Time series showing variation of the inverse ISI $\left(\Delta^{n}\right)^{-1}$ with $n$ for two particular values of $\alpha$.

be sensitive to the rise time of synaptic response even in the case of slow synaptic decay. In order to explore the distinct roles played by the rate of rise and fall of synaptic interactions, we generalize the alpha function of equation 3.3 by considering the difference of exponentials

$$
J(t)=\frac{\alpha_{1} \alpha_{2}}{\alpha_{2}-\alpha_{1}}\left(\mathrm{e}^{-\alpha_{1} t}-\mathrm{e}^{-\alpha_{2} t}\right) \Theta(t) .
$$

In the limit $\alpha_{2} \rightarrow \alpha_{1} \rightarrow \alpha$, this reduces to the alpha function (see equation 3.3). If $\alpha_{1}>\alpha_{2}$, then the asymptotic behavior of a synapse is approximately given by $\mathrm{e}^{-\alpha_{2} t}$, and the time to peak is $t_{p}=\left[\alpha_{1}-\alpha_{2}\right]^{-1} \ln \left(\alpha_{1} / \alpha_{2}\right)$. The roles of $\alpha_{1}$ and $\alpha_{2}$ are reversed if $\alpha_{2}>\alpha_{1}$.

In Figure 14a, we plot the critical coupling for a discrete Hopf bifurcation in the firing times for the excitatory-inhibitory pair previously analyzed in section 4.4. Now, however, the delay distribution $J(t)$ is taken to be given by equation 4.20 , with $\alpha_{2}=0.2$ and $\alpha_{1}$ a free parameter. Since the synaptic decay time is relatively slow, we expect there to be a good match between the IF and analog models. This is indeed found to be the case, as illustrated in Figure 14a, which shows that the critical coupling for bursting in the analog and IF models is in very close agreement. More surprising, it is seen that bursting persists even for large values of $\alpha_{1}$, that is, for fast synaptic rise times. (Bursting still occurs even when $\alpha_{1}$ is of the order 100 or more.) One might still expect the frequency of the oscillations to approach zero 

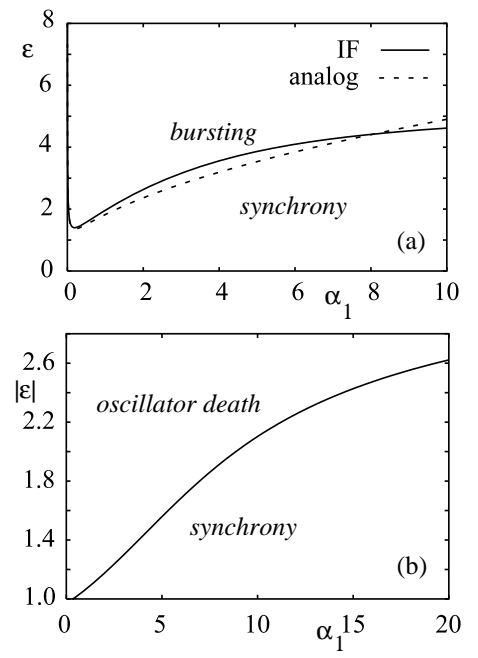

Figure 14: Critical coupling for a Hopf bifurcation in the firing times as a function of the rise time $\alpha_{1}$ for fixed decay time $\alpha_{2}=0.2$. (a) Excitatory-inhibitory pair with $\epsilon>0, W_{11}=W_{22}=0, W_{21}=1$, and $W_{12}=-2$. Bursting can occur even for fast rise times. The corresponding critical coupling for the analog model is shown as a dashed curve. (b) Inhibitory pair of IF neurons with $\epsilon<0$, $W_{11}=W_{22}=0, W_{21}=W_{12}=1$. Critical coupling is only weakly dependent on $\alpha_{1}$.

in the limit $\alpha_{1} \rightarrow \infty$. However, this is not found to be the case, which can be understood from the fact that the Hopf bifurcations are subcritical (cf. Figure $4 \mathrm{~b}$ ). Finally, in Figure $14 \mathrm{~b}$ we plot the critical coupling for oscillator death in an inhibitory pair of IF neurons. There is only a weak dependence on $\alpha_{1}$, which is consistent with the analog model.

\section{Discussion}

We have presented a dynamical theory of spiking neurons that bridges the gap between weakly coupled phase oscillator models and strongly coupled firing-rate (analog) models. The basic results of our article can be summarized as follows:

1. A fundamental mechanism for the desynchronization of IF neurons with strong synaptic coupling is a discrete Hopf bifurcation in the firing times. This typically leads to nonphase-locked behavior characterized by periodic or quasiperiodic variations of the ISIs on closed orbits. In the case of slow synapses, the coarse-grained dynamics can 
be represented very well by a corresponding analog model in which the output of a neuron is taken to be a mean firing rate.

2. A homogeneous network of $N$ synchronized IF neurons with global inhibitory coupling can destabilize in the strong coupling regime to a state in which some of the neurons became inactive (oscillator death). Thus the stability criterion obtained by van Vreeswijk et al. (1994) for $N=2$ is valid only in the weak coupling regime. There exists a critical value of the inverse rise time, $\alpha_{0}(N)$, such that synchrony persists for arbitrarily large coupling when $\alpha>\alpha_{0}(N)$ (fast synapses). Moreover, $\alpha_{0}(N) \rightarrow 0$ as $N \rightarrow \infty$. This establishes that the stability condition specified in the mode-locking theorem of Gerstner et al. (1996) is valid in the thermodynamic limit but no longer holds for finite networks with slow synapses.

3. Rhythmic bursting patterns can occur in asymmetric IF networks with a mixture of inhibitory and excitatory synaptic coupling. The ISIs evolve on periodic orbits that are large relative to the mean ISI within a burst. There is good agreement between the temporal variation of the ISIs in the IF model and the variation of the firing rate in the corresponding analog model.

4. A network of IF neurons with long-range interactions can desynchronize to a state with a regular spatial variation in the mean firing rate that is in good agreement with the corresponding analog model. The ISIs evolve on closed orbits that are well separated in phase-space. The fine temporal structure of the IF model leads to deterministic fluctuations of the activity patterns whose coefficient of variation is an increasing function of the inverse rise time $\alpha$. In other words, fast synapses lead to higher levels of deterministic noise.

An important question that we hope to address elsewhere concerns the extent to which the complex behavior exhibited by the IF networks studied in this article is mirrored by more biophysically detailed models of spiking neurons. Preliminary numerical studies of Hodgkin-Huxley neurons suggest a strong connection between the two classes of model. (See also White et al., 1998.) The advantage of working with the IF model is that it allows precise analytical statements to be made. Moreover, there are a number of ways of extending our analysis to take into account additional aspects of neuronal processing.

The effects of diffusion along the dendritic tree of a neuron can be incorporated into the model by taking $J(\tau)$ in equation 3.2 to be the Green's function of the corresponding cable equation. It is also possible to take into account active membrane properties under the assumption that variations of the dendritic membrane potential are small so that the channel kinetics can be linearized along the lines developed by Koch (1984). The resulting membrane impedance of the dendrites displays resonant-like behavior due to the 
additional presence of inductances. This leads to a corresponding form of resonant-like synchronization. In other words, there is a strongly enhanced (reduced) tendency to synchronize for excitatory (inhibitory) coupling when the resonant frequency of the dendritic membrane approximately matches the frequency of the oscillators. Active dendrites can also desynchronize homogeneous networks of IF oscillators with strong excitatory or inhibitory coupling, leading to periodic bursting patterns (Bressloff, 1999).

The response of a network to a sinusoidal external stimulus may be studied by taking $I_{i}=A+B \sin (\omega t)$ in equation 3.1, where $A, B$ are constants. Since the resulting dynamical system is now nonautonomous, it is no longer possible to specify phase locking in terms of $N-1$ relative phases and a self-consistent collective period (see equation 4.1). However, one can derive conditions under which the network is entrained to the external stimulus. For example, in the case of 1:1 mode locking, the firing times of the IF neurons are of the form $T_{j}^{m}=\left(m-\phi_{j}\right) T$, with $T=2 \pi / \omega$. The absolute phases $\phi_{j}$ are determined from the set of equations

$$
1=\left(1-\mathrm{e}^{-T}\right) A+\left(1-\mathrm{e}^{-T}\right) F\left(\phi_{i}\right)+\epsilon \sum_{j=1}^{N} W_{i j} K_{T}\left(\phi_{j}-\phi_{i}\right),
$$

with $K_{T}$ given by equation 4.2 and $F(\phi)=B[\sin (2 \pi \phi)+\omega \cos (2 \pi \phi)] /\left(1+\omega^{2}\right)$. It is also possible to investigate the stability of such solutions by a relatively straightforward generalization of the analysis presented in section 4.2. Another interesting extension is to $p: q$ mode locking. For example, suppose that the neurons have $T$-periodic firing patterns in which the $j$ th neuron fires $q_{j}$ times over an interval $T$. In order to generate such solutions, it is necessary to take the firing times to have the general form (Coombes \& Bressloff, 1999):

$$
T_{j}^{n}=T\left(\left[\frac{n}{q_{j}}\right]-\phi_{j, n} \bmod q_{j}\right),
$$

where [.] denotes the integer part. This generates a set of $\sum_{j=1}^{N} q_{j}$ closed equations for the absolute phases $\phi_{j, n} \bmod q_{j}$. One can use these equations to determine the borders in parameter space where the ansatz 5.2 for mode locking fails. The set of such borders will define an Arnold tongue diagram, with overlapping tongues indicating possible regions of multistability and chaos. Similar techniques can be used to study the bursting solutions found in section 4.4. The existence of chaotic solutions can be studied using an extension of the notion of a Lyapunov exponent that takes into account the presence of discontinuities in the dynamics due to reset (Coombes \& Bressloff, 1999). Incidentally, the construction of a Lyapunov exponent also allows one to understand the spike-time reliability of an IF neuron in response to small, aperiodic signals as recently observed by Hunter, Milton, Thomas, \& Cowan (1998). 
Another important issue concerns the effects of noise. We expect that sufficiently small levels of noise will not alter the basic results of this article. On the other hand, the presence of noise can lead to new and interesting phenomena as illustrated by the numerical study of pattern formation by Usher et al. (1994) and Usher, Stemmler, \& Olami (1995). They considered a two-dimensional network of IF neurons with short-range excitation and long-range inhibition, fast synapses, and a stochastic external input. They observed patterns of activity in the mean firing rate that are two-dimensional versions of the patterns shown in the inset of Figure 12. It was found that in a certain parameter regime, these patterns were metastable in the presence of noise and tended to diffuse through the network. This macroscopic dynamics resulted in $1 / f$ power spectra and power law distributions of the ISIs on the microscopic scale of a single neuron, and led to a large coefficient of variation, $C_{V}>1$. We are currently carrying out a detailed study of pattern formation in two-dimensional IF networks using the methods presented in our article and hope to gain further insight into the observations of Usher et al. (1994, 1995), in particular, the interplay between noise and the underlying deterministic dynamics. One way of including noise in the IF model or the spike-response model is by introducing a probability $P$ of firing during an infinitesimal time $\delta t$ : $P(U ; \delta t)=\tau^{-1}(U) \delta t$. To mimic more realistic models, the response time $\tau(U)$ is chosen to be large if $U<h$ and to vanish if $U \gg h$. This is achieved by writing $\tau(U)=\tau_{0} \mathrm{e}^{-\beta(U-h)}$, where $\tau_{0}$ is the response time at threshold and $\beta$ determines the level of noise. The deterministic case is recovered in the limit $\beta \rightarrow \infty$. In the case of large, homogeneous networks, one can use a meanfield approach to reduce the dynamics to an analytically tractable form (see Gerstner, 1995). Note that another consequence of noise is to smooth out the firing-rate function (see equation 3.18) in the analog version of the model.

A final aspect we would like to highlight here is the distinction between excitable and oscillatory networks. In this article we have focused on the latter case by taking $I_{i}>1$ in equation 3.1 so that the network may be viewed as a coupled system of oscillators with natural periods $T_{i}=\ln \left[I_{i} /\left(I_{i}-1\right)\right]$. However, it is also possible for spontaneous network oscillations to occur in the excitable regime, whereby $I_{i}<1$ for all $i=1, \ldots, N$. All of the basic analytical results presented in section 4 can be carried over to the excitable regime, in particular, the phase-locking equation 4.1 and the eigenvalue equation 4.5. On the other hand, the weak coupling theory of section 3.1 is no longer applicable since one requires a finite level of coupling to maintain oscillatory behaviour. There have also been a number of studies of waves in excitable spiking networks in both one dimension (Ermentrout \& Rinzel, 1981; Ermentrout, 1998b) and two dimensions (Chu et al., 1994; Kistler et al., 1998). We are currently carrying out a detailed comparison of wave phenomena in excitable and oscillatory IF networks using some of the ideas presented in this article. 


\section{Acknowledgments}

We thank Jack Cowan at the University of Chicago for helpful discussions during the completion of this work. We are also grateful for the constructive comments of the referees and the suggestion to include the example in section 4.6. The research was supported by grant number GR/K86220 from the EPSRC (UK).

\section{References}

Abbott, L. F. (1991). Realistic synaptic inputs for model neural networks. Network, 2, 245-258.

Abbott, L. F. (1994). Decoding neuronal firing and modeling neural networks. Quart. Rev. Biophys., 27, 291-331.

Abbott, L. F., \& Kepler, T. B. (1990). Model neurons: From Hodgkin-Huxley to Hopfield. In Luis Garrido (Ed.), Statistical mechanics of neural networks. Berlin: Springer-Verlag.

Abbott, L. F., \& van Vreeswijk, C. (1993). Asynchronous states in neural networks of pulse-coupled oscillators. Phys. Rev. E., 48, 1483-1490.

Amit, D. J., \& Tsodyks, M. V. (1991). Quantitative study of attractor neural networks retrieving at low spike rates I: Substrate-spikes, rates and neuronal gain. Network, 2, 259-274.

Atiya, A., \& Baldi, P. (1989). Oscillations and synchronization in neural networks: An exploration of the labeling hypothesis. Int. J. Neural Syst., 1, 103-124.

Berry, M. J., Warland, D. K., \& Meister, M. (1997). The structure and precision of retinal spike trains. Proc. Natl. Acad. Sci. USA, 94, 5411-5416.

Bressloff, P. C. (1999). Resonant-like synchronization and bursting in a model of pulse-coupled neurons with active dendrites. J. Comp. Neurosci., 6, 237-249.

Bressloff, P. C., \& Coombes, S. (1997). Physics of the extended neuron. Int. J. Mod. Phys., 11, 2343-2392.

Bressloff, P. C., \& Coombes, S. (1998a). Travelling waves in chains of pulsecoupled oscillators. Phys. Rev. Lett., 80, 4185-4188.

Bressloff, P. C., \& Coombes, S. (1998b). Desynchronization, mode-locking and bursting in strongly coupled IF oscillators. Phys. Rev. Lett., 81, 2168-2171.

Bressloff, P. C., \& Coombes, S. (1999). Symmetry and phase-locking in a ring of pulse-coupled oscillators with distributed delays. Physica D, 126, 99-122.

Bressloff, P. C., Coombes, S., \& De Souza, B. (1997). Dynamics of a ring of pulsecoupled oscillators: A group theoretic approach. Phys. Rev. Lett., 79, 27912794.

Carr, C. E., \& Konishi, M. (1990). A circuit for detection of interaural differences in the brain stem of the barn owl. J. Neurosci., 10, 3227-3246.

Chow, C. C. (1998). Phase-locking in weakly heterogeneous neuronal networks. Physica D, 118, 343-370.

Chu, P. H., Milton, J. G., \& Cowan, J. D. (1994). Connectivity and the dynamics of integrate-and-fire neural networks. Int. J. Bif. Chaos, 4, 237-243.

Coombes, S., \& Bressloff, P. C. (1999). Mode-locking and Arnold tongues in 
periodically forced integrate-and-fire oscillators. Phys. Rev. E, 60, 2086-2096.

Coombes, S., \& Lord, G. J. (1997). Desynchronisation of pulse-coupled integrateand-fire neurons. Phys. Rev. E, 55, 2104-2107.

Crook, S. M., Ermentrout, G. B., Vanier, M. C., \& Bower, J. M. (1997). The role of axonal delay in the synchronization of networks of coupled cortical oscillators. J. Comp. Neurosci., 4, 161-172.

Destexhe, A., Mainen, Z. F., \& Sejnowski, T. J. (1994). Synthesis of models for excitable membranes synaptic transmission and neuromodulation using a common kinetic formalism. J. Comp. Neurosci., 1, 195-231.

Ermentrout, G. B. (1985). The behavior of rings of coupled oscillators. J. Mathemat. Biol., 23, 55-74.

Ermentrout, G. B. (1994). Reduction of conductance-based models with slow synapses to neural nets. Neural Computation, 8, 679-695.

Ermentrout, G. B. (1998a). Neural networks as spatial pattern forming systems. Rep. Prog. Phys., 61, 353-430.

Ermentrout, G. B. (1998b). The analysis of synaptically generated traveling waves. J. Comp. Neurosci., 5, 191-208.

Ermentrout, G. B., \& Cowan, J. D. (1979). A mathematical theory of visual hallucination pattens. Biol. Cybern., 34, 137-150.

Ermentrout, G. B., \& Kopell, N. (1984). Frequency plateaus in a chain of weakly coupled oscillators. SIAM J. Math. Anal., 15, 215-237.

Ermentrout, G. B., \& Rinzel, J. (1981). Waves in a simple excitable or oscillatory, reaction-diffusion model. J. Math. Biol., 11, 1269-294.

Ernst, U., Pawelzik, K., \& Giesel, T. (1995). Synchronization induced by temporal delays in pulse-coupled oscillators. Phys. Rev. Lett., 74(9), 1570-1573.

Gerstner, W. (1995). Time structure of the activity in neural-network models. Phys. Rev. E, 51(1), 738-758.

Gerstner, W., Kreiter, A. K., Markram, H., \& Herz, A .K. M. (1997). Neural codes: Firing rates and beyond. Proc. Natl. Acad. Sci. USA, 94, 12740-12741.

Gerstner, W., Ritz, R., \& van Hemmen, J. L. (1993). A biologically motivated and analytically soluble model of collective oscillations in the cortex I: Theory of weak locking. Biol. Cybern., 68, 363-374.

Gerstner, W., \& van Hemmen, J. L. (1994). Coding and information processing in neural networks. In E. Domany, J. L. van Hemmen, and K. Schulten (Eds.), Models of neural networks II (pp. 1-93). Berlin: Springer-Verlag.

Gerstner, W., van Hemmen, J. L., \& Cowan, J. D. (1996). What matters in neuronal locking. Neural Comput., 94, 1653-1676.

Golomb, D., \& Rinzel, J. (1994). Clustering in globally coupled inhibitory neurons. Physica D, 72, 259-282.

Han, S. K., Kurrer, C., \& Kuramoto, Y. (1995). Dephasing and bursting in coupled neural oscillators. Phys. Rev. Lett., 75, 3190-3193.

Hansel, D., Mato, G., \& Meunier, C. (1995). Synchrony in excitatory neural networks. Neural Comput., 7, 307-337.

Hopfield, J. J. (1984). Neurons with graded response have computational properties like those of two-state neurons. Proc. Natl. Acad. Sci. U.S.A., 81, 3088-3092.

Hopfield, J. J. (1995). Pattern recognition computation using action potential timing for stimulus representation. Nature, 376, 33-36. 
Hunter, J. D., Milton, J. G., Thomas, P. J., \& Cowan, J. D. (1998). A resonance effect for neural spike time reliability J. Neurophysiol., 80, 1427-1438.

Keener, J. P., Hoppensteadt, F. C., and Rinzel, J. (1981). Integrate-and-fire models of nerve membrane response to oscillatory input. SIAM J. Appl. Math., 41(3), 503-517.

Kepler, T. B., Abbott, L. F., and Marder, E. (1992). Reduction of conductancebased neuron models. Biological Cybernetics, 66, 381-387.

Kistler, W. M., Gerstner, W., \& van Hemmen, J. L. (1997). Reduction of the Hodgkin-Huxley equations to a single-variable threshold model. Neural Comput., 9, 1015-1045.

Kistler, W. M., Seitz, R., \& van Hemmen, J. L. (1998). Modeling collective excitations in cortical tissue. Physica D, 114, 273-295.

Koch, C. (1984). Cable theory in neurons with active, linearized membranes. Biol. Cybern., 50, 15-33.

Kuramoto, Y. (1984). Chemical oscillations, waves and turbulence. Berlin: SpringerVerlag.

Li, Z., \& Hopfield, J. J. (1989). Modeling the olfactory bulb and its neural oscillatory processings. Biol. Cybern., 61, 379-392.

Mainen, Z., \& Sejnowski, T. J. (1995). Reliability of spike timing in neocortical neurons. Science, 268, 1503-1506.

Marcus, C. M., \& Westervelt, R. M. (1989). Stability of analog neural networks with delay. Phys. Rev. A, 39, 347-365.

Mirollo, R. E., \& Strogatz, S. H. (1990). Synchronisation of pulse-coupled biological oscillators. SIAM J. Appl. Maths, 50, 1645-1662.

Mulloney, B., Perkel, D. H., \& Budelli, R. W. (1981). Motor-pattern production: Interaction of chemical and electrical synapses. Brain Research, 229, 25-33.

Rieke, F., Warland, D., van Steveninck, R. R. D., \& Bialek, W. (1996). Spikes: Exploring the neural code. Cambridge, MA: MIT Press.

Softky, W. R., \& Koch, C. (1992). Cortical cells should fire regularly, but do not. Neural Computation, 4, 643-645.

Somers, D. C., Nelson, S. B., \& Sur, M. (1995). An emergent model of orientation selectivity in cat visual cortical simple cells. J. Neurosci., 15, 5448-5465.

Strong, S. P., Koberle, R., van Steveninck, R. R. D., \& Bialek, W. (1998). Entropy and information in neural spike trains. Phys. Rev. Lett., 80, 197-200.

Treves, A. (1993). Mean-field analysis of neuronal spike dynamics. Network, 4, 259-284.

Tsodyks, M., Mitkov, I., \& Sompolinsky, H. (1993). Pattern of synchrony in inhomogeneous networks of oscillators with pulse interactions. Phys. Rev. Lett., $71,1280-1283$.

Tuckwell, H. C. (1988). Introduction to theoretical neurobiology (Vol. 1). Cambridge: Cambridge University Press.

Usher, M., Stemmler, M., Koch, C., \& Olami, Z. (1994). Network amplification of local fluctuations causes high spike rate variability, fractal firing patterns and oscillatory local field potentials. Neural Comput., 5, 795-835.

Usher, M., Stemmler, M., \& Olami, Z. (1995). Dynamic pattern formation leads to $1 / f$ noise in neural populations. Phys. Rev. Lett., 74, 326-329. 
van Vreeswijk, C. (1996). Partial synchronization in populations of pulsecoupled oscillators. Phys. Rev. E., 54(5), 5522-5537.

van Vreeswijk, C., Abbott, L. F., \& Ermentrout, G. B. (1994). When inhibition not excitation synchronizes neural firing. J. Comp. Neurosci., 1, 313-321.

Wang, X.-J., \& Rinzel, J. (1992). Alternating and synchronous rhythms in reciprocally inhibitory model neurons. Neural Comput., 4, 89-97.

Wang, X.-J., \& Rinzel, J. (1995). Oscillatory and bursting properties of neurons. In M. Arbib (Ed.), Handbook of brain theory and neural networks (pp. 686-691). Cambridge, MA: MIT Press.

White, J. A., Chow, C. C., Ritt, J., Soto-Trevino, C., \& Kopell, N. (1998). Synchronization and oscillatory dynamics in heterogeneous, mutually inhibited neurons. J. Comput. Neurosci., 5, 5-16.

Wilson, H. R., \& Cowan, J. D. (1972). Excitatory and inhibitory interactions in localized populations of model neurons. J. Biophys., 12, 1-24.

Wilson, H. R., \& Cowan, J. D. (1973). A mathematical theory of the functional dynamics of cortical and thalamic nervous tissue. Kybernetik, 13, 55-80.

Received August 27, 1998; accepted January 27, 1999. 